\title{
Comprehensive Investigation of the Photophysical Properties of Alkynylcoumarin Gold(I) Complexes
}

Andrea Pinto, ${ }^{\mathrm{a}, \mathrm{b}}$ Carla Cunha, ${ }^{\mathrm{c}}$ Gabriel Aullón, ${ }^{\mathrm{a}, \mathrm{d}}$ João Carlos Lima, ${ }^{\mathrm{e}}$ Laura Rodríguez, ${ }^{\mathrm{a}, \mathrm{b} *}$ and J. Sérgio Seixas de Melo, ${ }^{\text {c* }}$

${ }^{a}$ Departament de Química Inorgànica i Orgànica, Secció de Química Inorgànica, Universitat de Barcelona, Martí i Franquès 1-11, E-08028 Barcelona, Spain

${ }^{b}$ Institut de Nanociència i Nanotecnologia (IN2UB). Universitat de Barcelona, 08028 Barcelona, Spain

${ }^{c}$ University of Coimbra, CQC, Department of Chemistry, Rua Larga, Coimbra3004-535, Portugal.

${ }^{d}$ Institut de Química Teòrica i Computacional (IQTCUB). Universitat de Barcelona, 08028 Barcelona, Spain

${ }^{e}$ LAQV-REQUIMTE, Departamento de Química, Universidade Nova de Lisboa, 2829-516 Monte de Caparica, Portugal

Email: sseixas@ci.uc.pt (J. Sérgio Seixas de Melo); laura.rodriguez@qi.ub.es (Laura Rodríguez)

\section{Supporting Information}




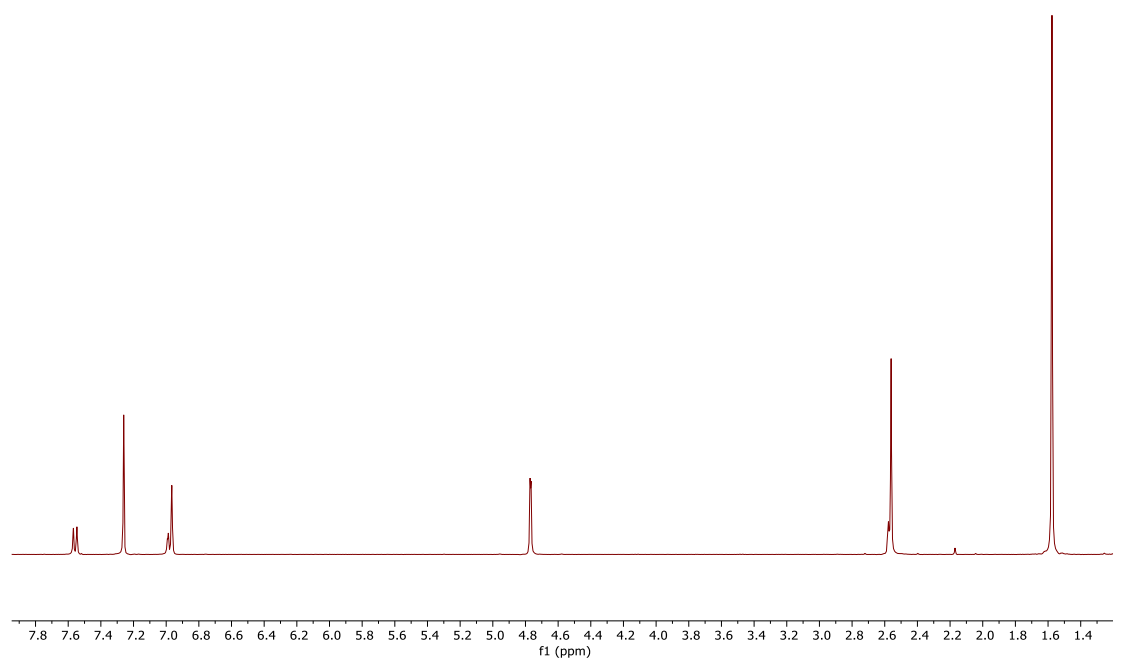

Figure S1. ${ }^{1} \mathrm{H}-\mathrm{NMR}$ of $\mathbf{3}$.

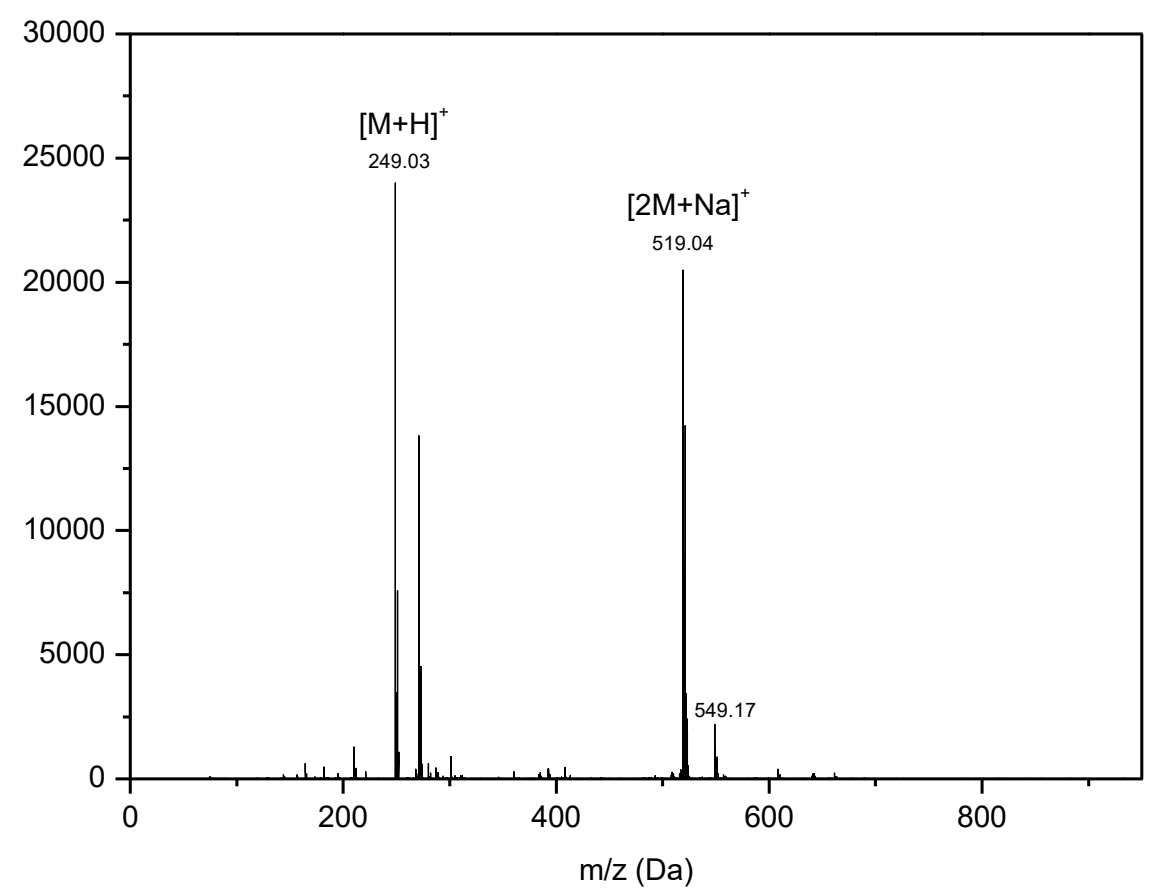

Figure S2. ESI-MS(+) spectrum of 3. 
Synthesis of [Au\{3-chloro-4-methyl-7-(prop-2-in-1-yloxy)-1-benzopyran-2-one\}-(PTA)] (3a). A solution of $\mathrm{KOH}(10.4 \mathrm{mg}, 0.18 \mathrm{mmol})$ in methanol $(2 \mathrm{~mL})$ was added to a solution of 3-chloro4-methyl-7-(prop-2-in-1-yloxy)-1-benzopyran-2-one (25 mg, $0.11 \mathrm{mmol})$ in methanol (5 mL). After $30 \mathrm{~min}$ of stirring a dichloromethane solution $(5 \mathrm{~mL})$ of [AuCl(PTA)] (41.5 mg, $0.11 \mathrm{mmol})$ was added and the solution was maintained at room temperature protected from the light with aluminium foil. After $1 \mathrm{~h}$ of stirring, the solution was concentrated to $c a .2 \mathrm{~mL}$ and hexane $(5 \mathrm{~mL})$ was added to precipitate an orange pale solid which was filtered and obtained in $47 \%$ yield (30 $\mathrm{mg})$.

${ }^{1} \mathrm{H}-\mathrm{NMR}\left(\mathrm{CD}_{3} \mathrm{CN}, \mathrm{ppm}\right): 7.67$ (d, J = $\left.8.8 \mathrm{~Hz}, 1 \mathrm{H}, \mathrm{O}-\mathrm{C}-\mathrm{CH}-\mathrm{CH}\right), 6.99$ (d, J = 8.8 Hz, 1H, O-C$\mathrm{CH}-\mathrm{CH}), 6.93(\mathrm{~s}, 1 \mathrm{H}, \mathrm{CO}-\mathrm{CH}-\mathrm{C}), 4.81\left(\mathrm{~s}, 2 \mathrm{H}, \mathrm{CH}_{2}\right), 4.52-4.36\left(\mathrm{AB} \mathrm{q}, \mathrm{J}=13 \mathrm{~Hz}, 6 \mathrm{H}, \mathrm{N}-\mathrm{CH}_{2}-\right.$ $\mathrm{N}), 4.19$ (s, 6H, N-CH $2-\mathrm{P}), 2.54$ (s, 3H, CH 3$) .{ }^{31} \mathrm{P}\left\{{ }^{1} \mathrm{H}\right\}-\mathrm{NMR}\left(\mathrm{CD}_{3} \mathrm{CN}, \mathrm{ppm}\right):-49.9$. IR $\left(\mathrm{KBr}, \mathrm{cm}^{-}\right.$ $\left.{ }^{1}\right)$ : $2100(\mathrm{C} \equiv \mathrm{C}), 1670(\mathrm{C}=\mathrm{O})$. ESI-MS $(+) \mathrm{m} / z$ : $602.0667\left(\left[\mathrm{M}+\mathrm{H}^{+}\right]^{+}\right.$, calc: 602.0596).

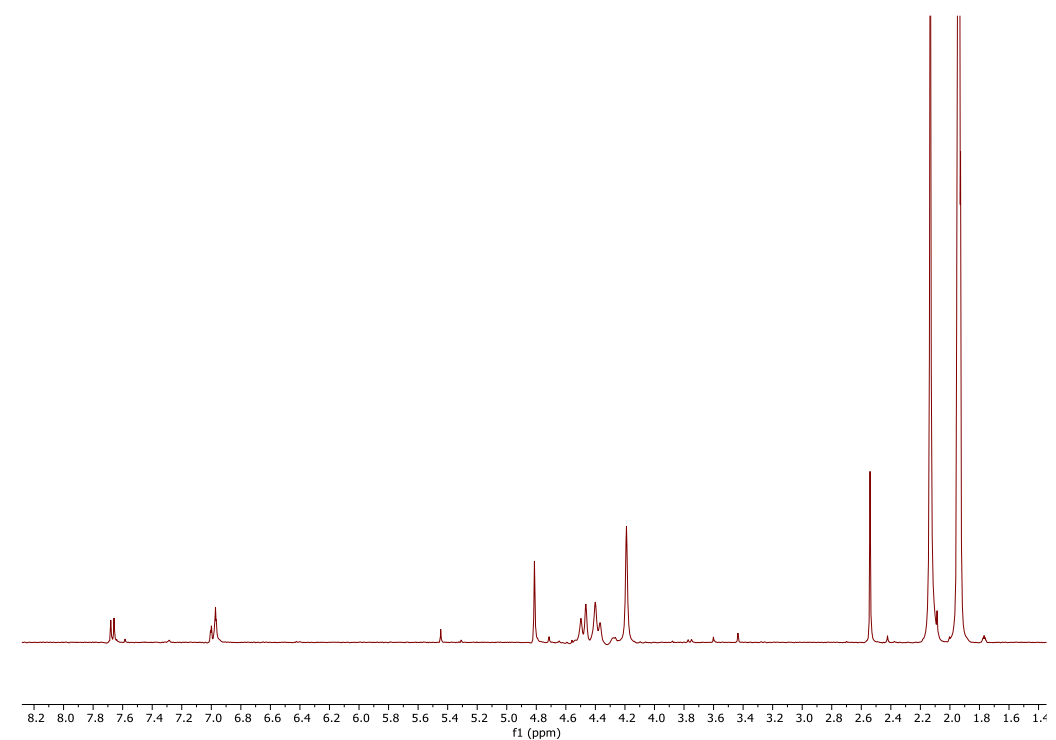

Figure S3. ${ }^{1} \mathrm{H}$ NMR spectrum of $\mathbf{3 a}$. 


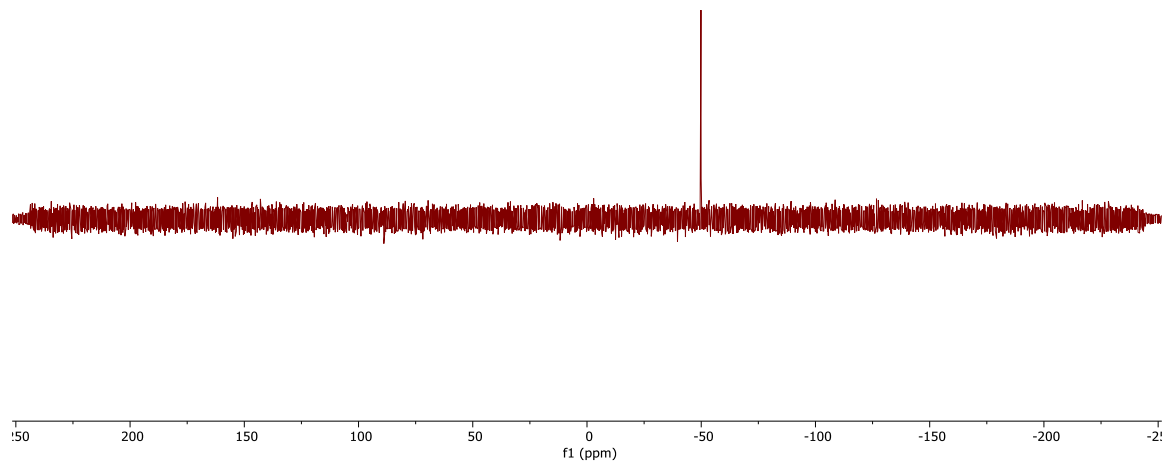

Figure S4. ${ }^{31} \mathrm{P}$ NMR spectrum of $\mathbf{3 a}$.

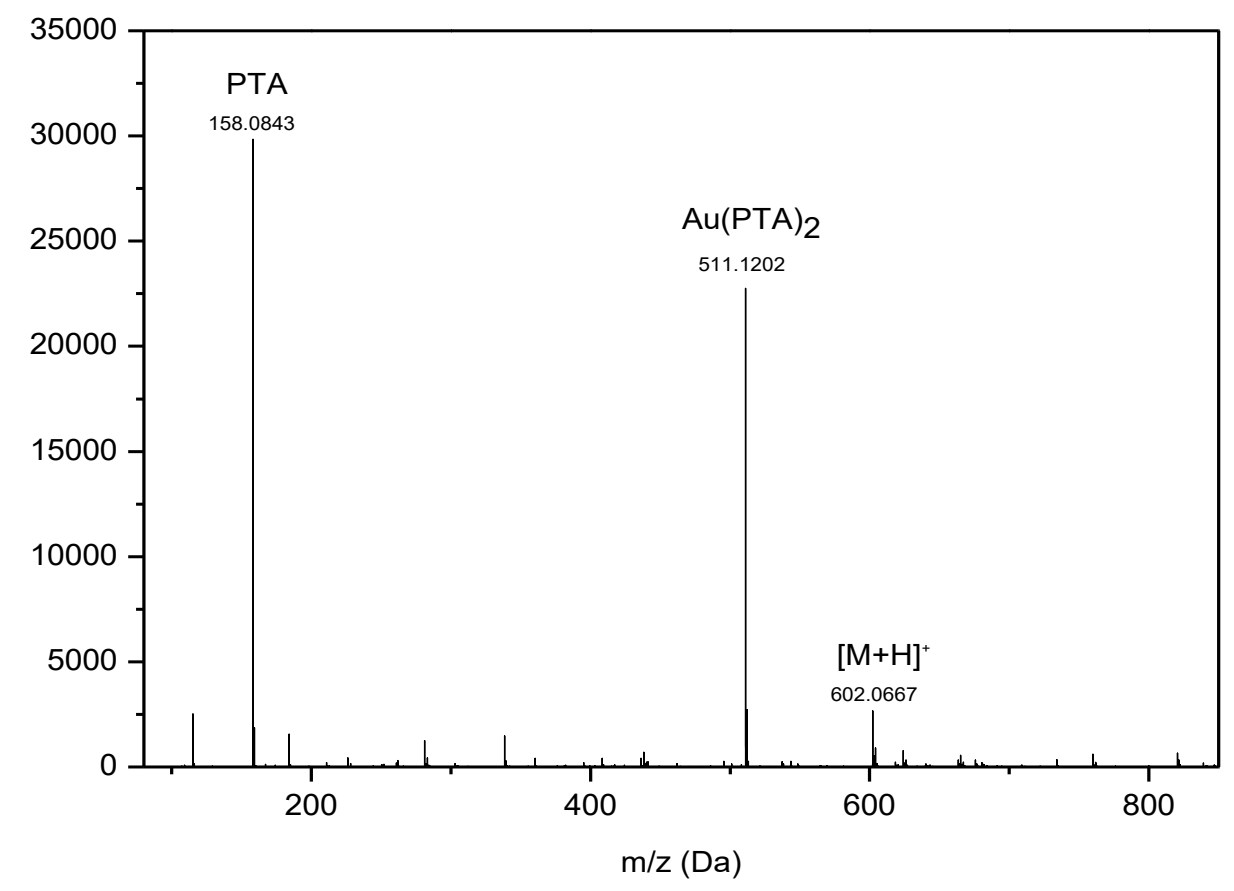

Figure S5. ESI-MS(+) spectrum of 3a. 
Synthesis of [Au\{3-chloro-4-methyl-7-(prop-2-in-1-yloxy)-1-benzopyran-2-one\}-(DAPTA)] (3b). Details of synthesis 3a were also applied to the preparation of this compound but using DAPTA instead of PTA. A pale orange solid was obtained in $69 \%$ yield $(50 \mathrm{mg})$.

${ }^{1} \mathrm{H}-\mathrm{NMR}\left(\mathrm{CD}_{3} \mathrm{CN}, \mathrm{ppm}\right): 7.51(\mathrm{~d}, \mathrm{~J}=8.8 \mathrm{~Hz}, 1 \mathrm{H}, \mathrm{O}-\mathrm{C}-\mathrm{CH}-\mathrm{CH}), 7.10(\mathrm{~s}, 1 \mathrm{H}, \mathrm{CO}-\mathrm{CH}-\mathrm{C}), 6.96$ $(\mathrm{dd}, \mathrm{J}=8.8 \mathrm{~Hz}, 1 \mathrm{H}, \mathrm{O}-\mathrm{C}-\mathrm{CH}-\mathrm{CH}), 5.78\left(\mathrm{~d}, \mathrm{~J}=14 \mathrm{~Hz}, 1 \mathrm{H}, \mathrm{N}-\mathrm{CH}_{2}-\mathrm{N}\right), 5.58\left(\mathrm{~m}, 1 \mathrm{H}, \mathrm{N}-\mathrm{CH}_{2}-\mathrm{P}\right)$, $4.95\left(\mathrm{~d}, \mathrm{~J}=14 \mathrm{~Hz}, 1 \mathrm{H}, \mathrm{N}-\mathrm{CH}_{2}-\mathrm{N}\right), 4.86\left(\mathrm{~s}, 2 \mathrm{H}, \mathrm{CH}_{2}\right), 4.66-4.56\left(\mathrm{~m}, 2 \mathrm{H}, \mathrm{N}-\mathrm{CH}_{2}-\mathrm{N}+\mathrm{N}_{-}-\mathrm{CH}_{2}-\mathrm{P}\right)$, 4.09-4.03 (m, 2H, N-CH $\left.-\mathrm{N}+\mathrm{N}-\mathrm{CH}_{2}-\mathrm{P}\right), 3.81$ (s, 2H, N-CH $-\mathrm{P}$ ), 3.46 (d, J = $16 \mathrm{~Hz}, 1 \mathrm{H}, \mathrm{N}-\mathrm{CH}_{2}-$ $\mathrm{P}), 2.55$ (s, 3H, $\left.\mathrm{CH}_{3}\right), 2.10$ (s, 6H, N-CO-CH$)_{3} .{ }^{31} \mathrm{P}\left\{{ }^{1} \mathrm{H}\right\}-\mathrm{NMR}\left(\mathrm{CD}_{3} \mathrm{CN}, \mathrm{ppm}\right)$ : - 22.4. IR (KBr, $\left.\mathrm{cm}^{-1}\right): 2110(\mathrm{C} \equiv \mathrm{C}), 1622(\mathrm{C}=\mathrm{O})$. ESI-MS $(+) \mathrm{m} / z: 674.0877\left(\left[\mathrm{M}+\mathrm{H}^{+}\right]^{+}\right.$, calc: 674.0803).

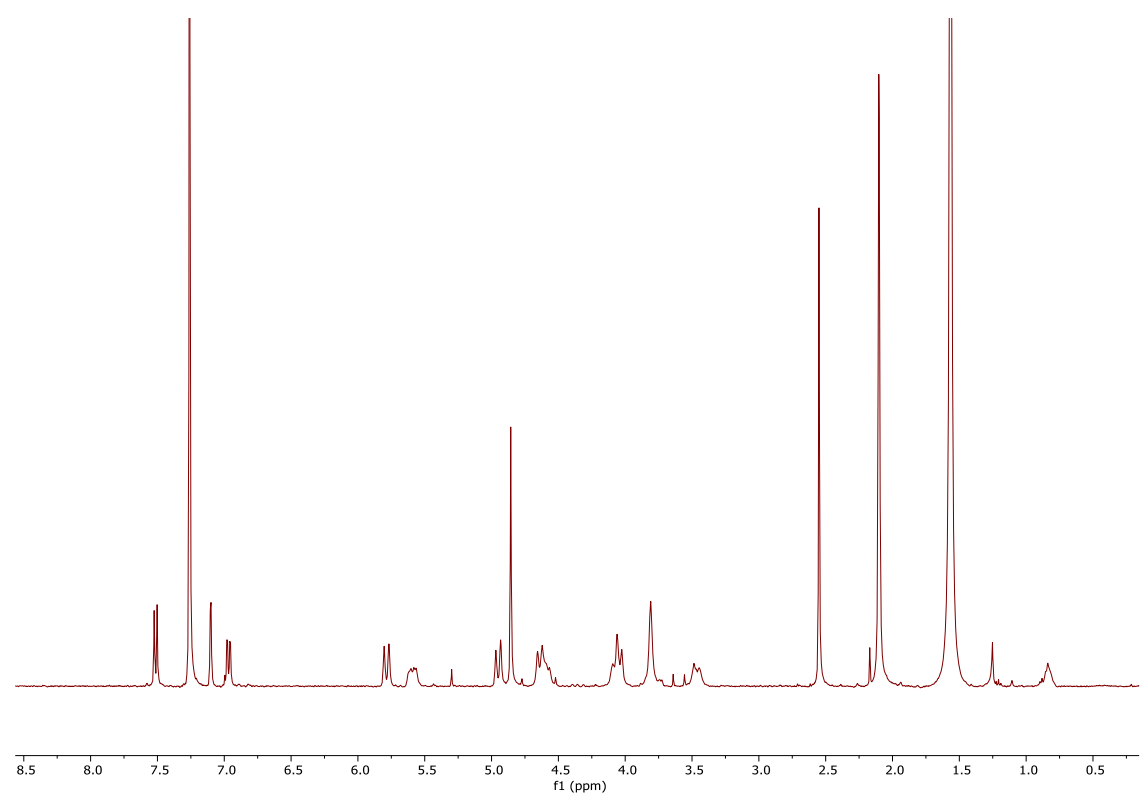

Figure S6. ${ }^{1} \mathrm{H}$ NMR spectrum of $\mathbf{3 b}$.

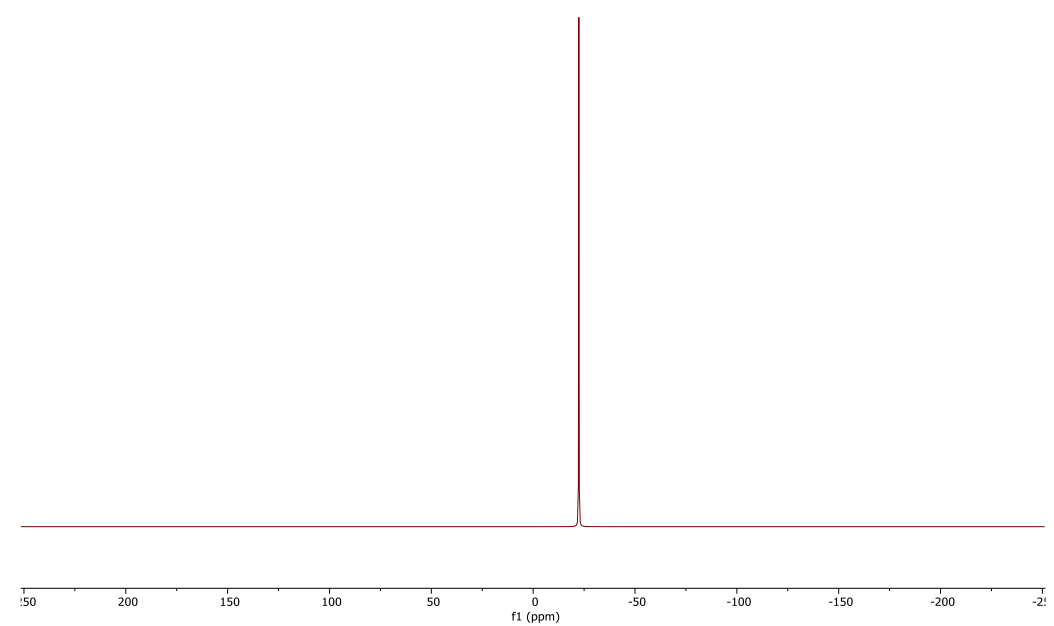

Figure S7. ${ }^{31} \mathrm{P}$ NMR spectrum of $\mathbf{3 b}$. 


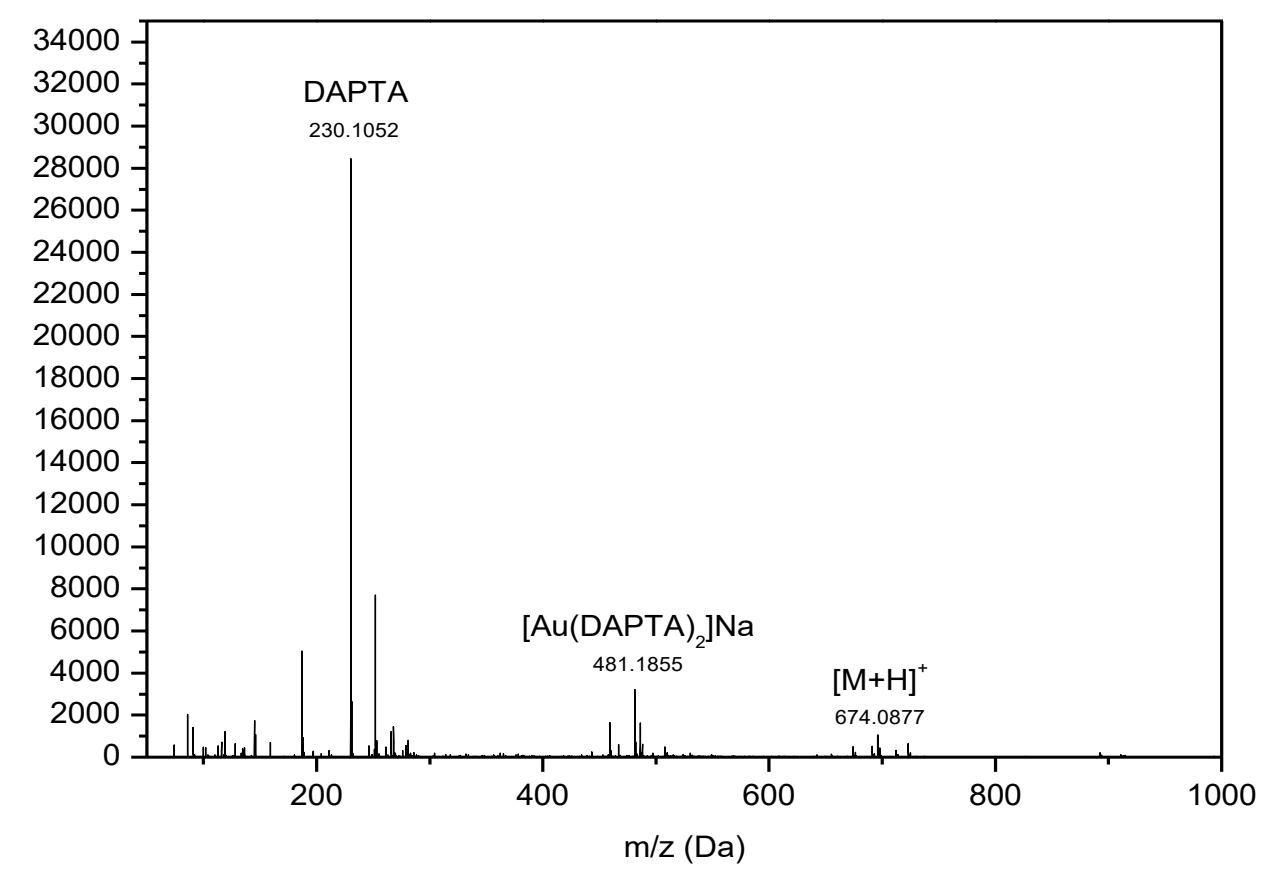

Figure S8. ESI-MS(+) spectrum of $\mathbf{3 b}$. 
Table S1. Calculated absorption parameters (wavelengths in $\mathrm{nm}$ and their intensities) for organic coumarins in gas phase and acetonitrile solution. For each entry, main contributions of the orbitals for the transition and their coefficients are shown.

(a) Ligand 1

\begin{tabular}{|c|c|c|c|}
\hline \multicolumn{2}{|r|}{ Gas phase } & \multicolumn{2}{|r|}{$\mathrm{CH}_{3} \mathrm{CN}$} \\
\hline$\lambda(f)$ & Assignment & $\lambda(f)$ & Assignment \\
\hline $303(0.35)$ & $\begin{array}{l}\text { Coumarin: } \\
\pi(\mathrm{Cou}) \rightarrow \pi^{*}(\mathrm{Cou})[94 \%]\end{array}$ & $308(0.47)$ & $\begin{array}{l}\text { Coumarin: } \\
\pi(\mathrm{Cou}) \rightarrow \pi^{*}(\mathrm{Cou})[96 \%]\end{array}$ \\
\hline
\end{tabular}

(b) Ligand 2

\begin{tabular}{|c|c|c|c|}
\hline \multicolumn{2}{|r|}{ Gas phase } & \multicolumn{2}{|r|}{$\mathrm{CH}_{3} \mathrm{CN}$} \\
\hline$\lambda(f)$ & Assignment & $\lambda(f)$ & Assignment \\
\hline $300(0.33)$ & $\begin{array}{l}\text { Coumarin: } \\
\pi(\mathrm{Cou}) \rightarrow \pi^{*}(\mathrm{Cou})[93 \%]\end{array}$ & $305(0.44)$ & $\begin{array}{l}\text { Coumarin: } \\
\pi(\mathrm{Cou}) \rightarrow \pi^{*}(\mathrm{Cou})[96 \%]\end{array}$ \\
\hline
\end{tabular}

(c) Ligand 3

\begin{tabular}{|c|c|c|c|}
\hline \multicolumn{2}{|r|}{ Gas phase } & \multicolumn{2}{|r|}{$\mathrm{CH}_{3} \mathrm{CN}$} \\
\hline$\lambda(f)$ & Assignment & $\lambda(f)$ & Assignment \\
\hline $310(0.41)$ & $\begin{array}{l}\text { Coumarin: } \\
\pi(\mathrm{Cou}) \rightarrow \pi^{*}(\mathrm{Cou})[95 \%]\end{array}$ & $317(0.49)$ & $\begin{array}{l}\text { Coumarin: } \\
\pi(\mathrm{Cou}) \rightarrow \pi^{*}(\mathrm{Cou})[97 \%]\end{array}$ \\
\hline
\end{tabular}




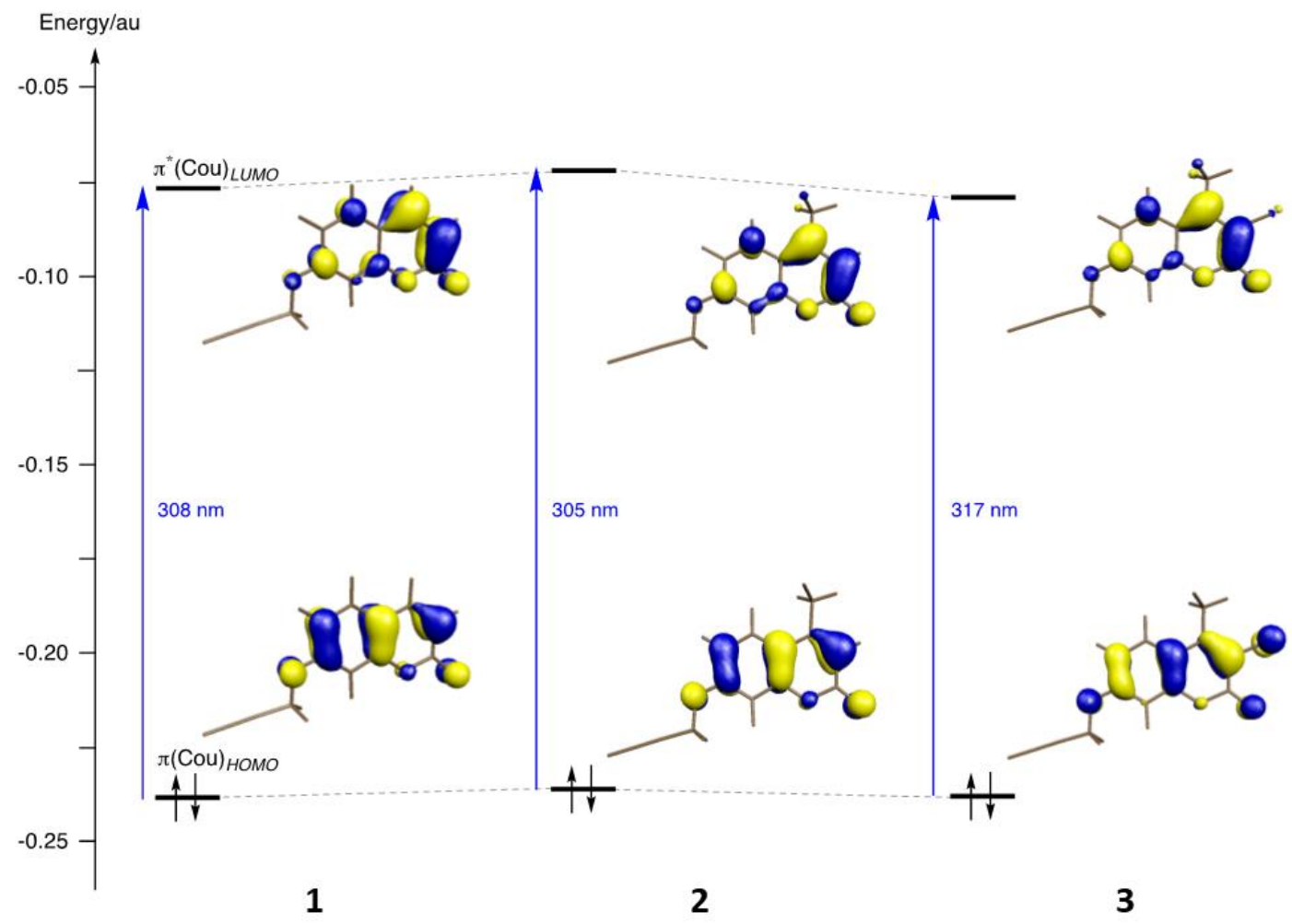

Figure S9. Schematic representation of main expected transition in the absorption spectra of organic coumarins (1-3). 
(a) ligand 1

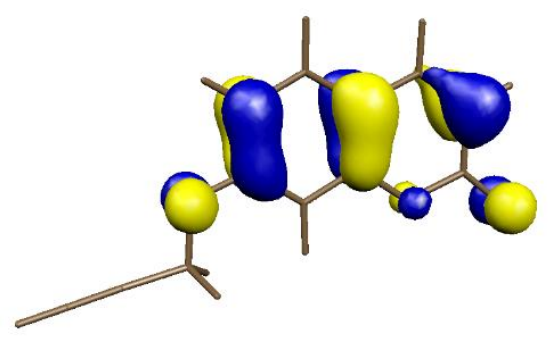

MO 52 (HOMO): $-0.239 \mathrm{Ha}$

Cou (86), $\mathrm{OCH}_{2}(10)$

(b) ligand 2

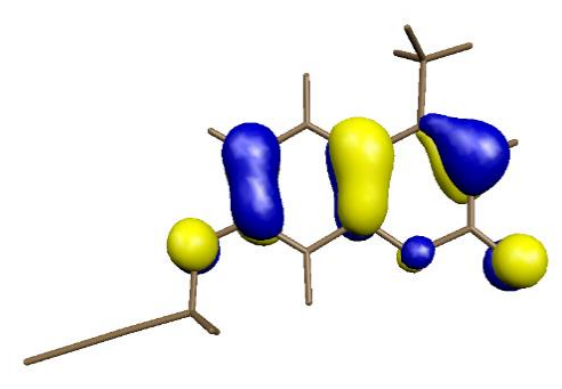

MO 56 (HOMO): $-0.236 \mathrm{Ha}$

Cou (86), $\mathrm{OCH}_{2}(10)$

(c) ligand $\mathbf{3}$

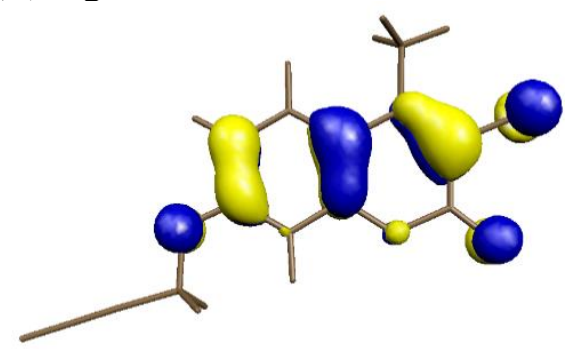

MO 64 (HOMO): $-0.238 \mathrm{Ha}$

Cou (86), $\mathrm{OCH}_{2}(9)$

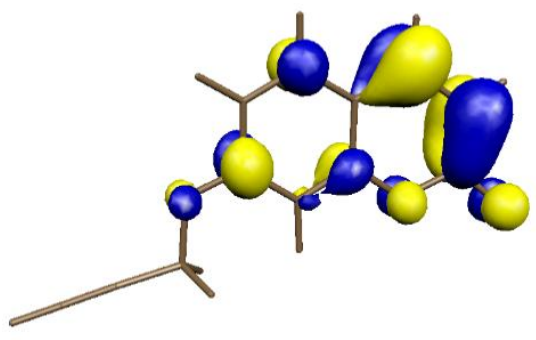

MO 53 (LUMO): $-0.077 \mathrm{Ha}$ Cou (93)

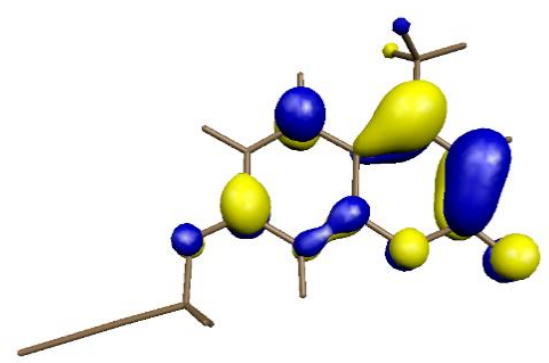

MO 57 (LUMO): -0.072 Ha Cou (94)

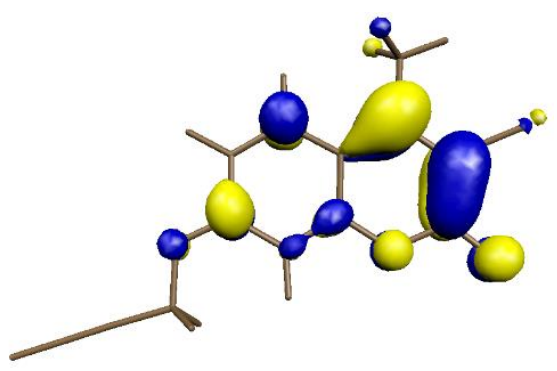

MO 65 (LUMO): $-0.079 \mathrm{Ha}$ Cou (95)

Figure S10. Molecular orbitals for ligands 1-3. Percentage compositions for each one entry from Natural Populations Analysis are shown. 


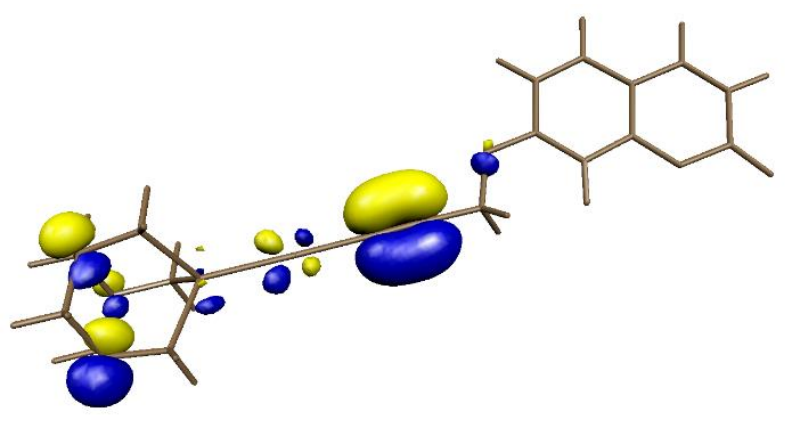

MO 99: $-0.253 \mathrm{Ha}$

CC (54), PTA (34)

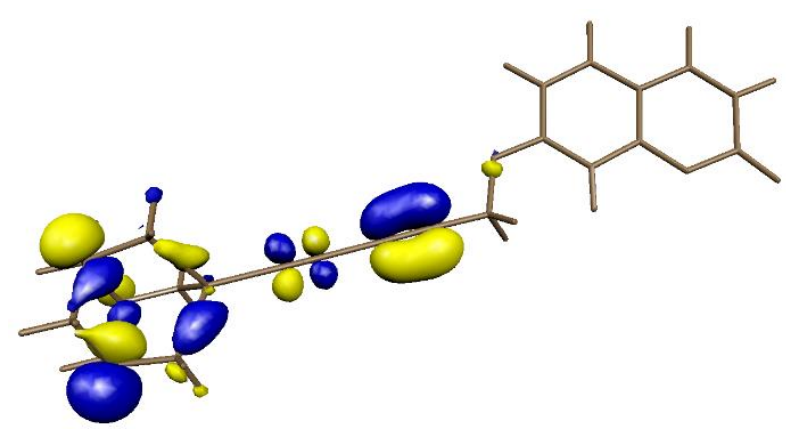

MO 101: $-0.241 \mathrm{Ha}$

PTA (55), CC (30), Au (7)

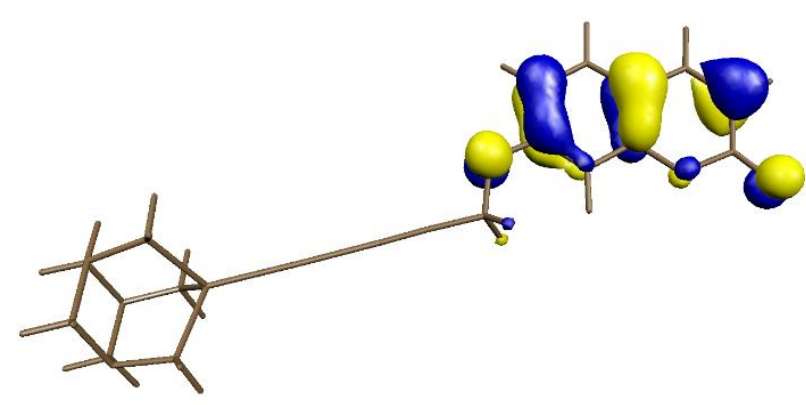

MO 103 (HOMO): $-0.223 \mathrm{Ha}$

Cou (81), $\mathrm{OCH}_{2}(14)$

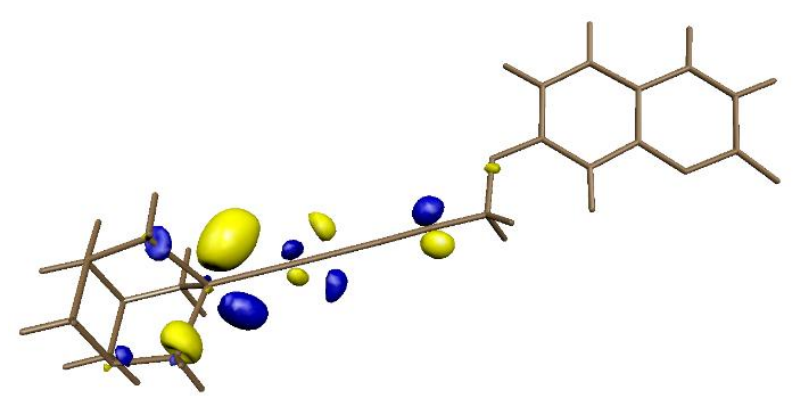

MO 105: $-0.040 \mathrm{Ha}$

PTA (48), Au (42), CC (8)

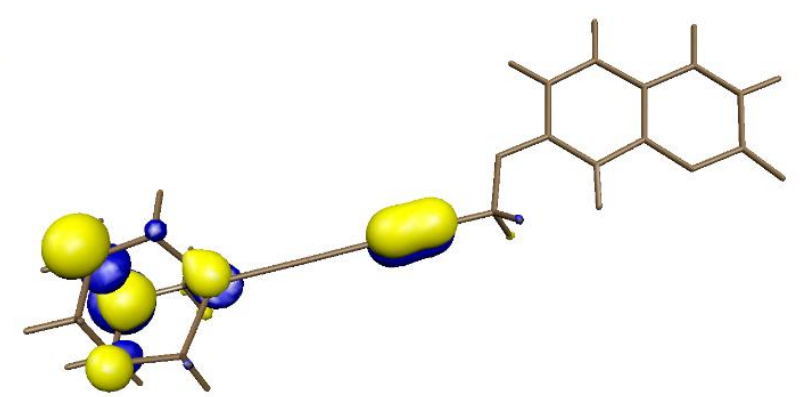

MO 100: $-0.248 \mathrm{Ha}$

PTA (68), CC (21)

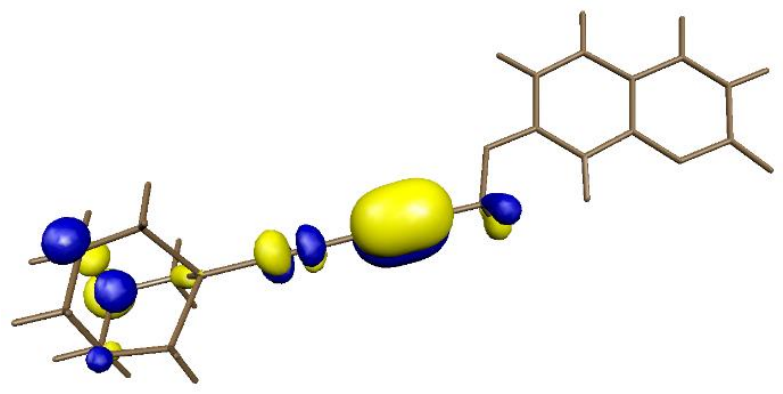

MO 102: $-0.235 \mathrm{Ha}$

CC (59), PTA (21), Au (8), $\mathrm{OCH}_{2}$ (4)

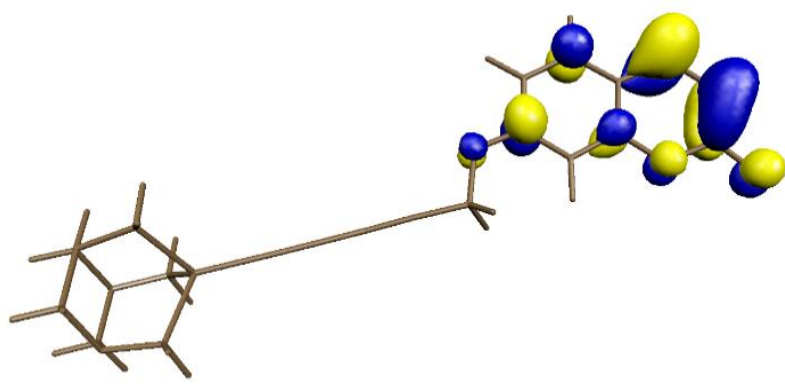

MO 104 (LUMO): $-0.064 \mathrm{Ha}$

Cou (94)

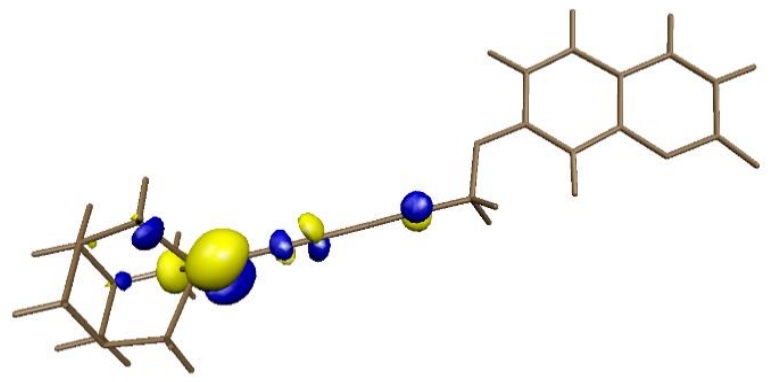

MO 106: $-0.038 \mathrm{Ha}$

PTA (48), Au (42), CC (5)

Figure S11. Molecular orbitals for complex 1a. Percentage compositions for each one entry from Natural Populations Analysis are shown. 


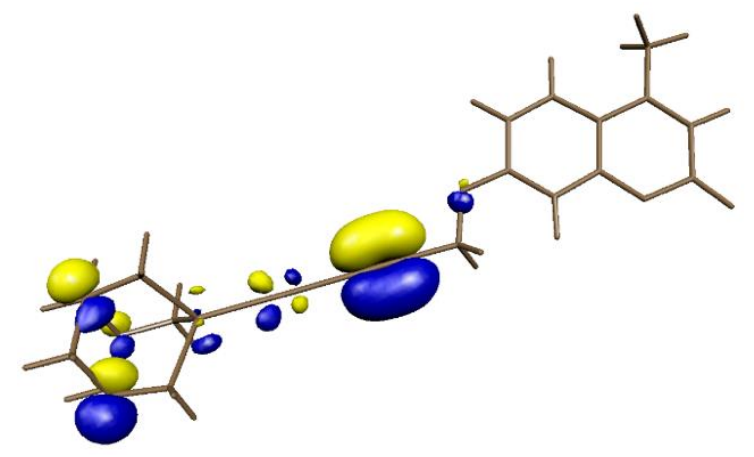

MO 103: $-0.252 \mathrm{Ha}$

CC (52), PTA (36)

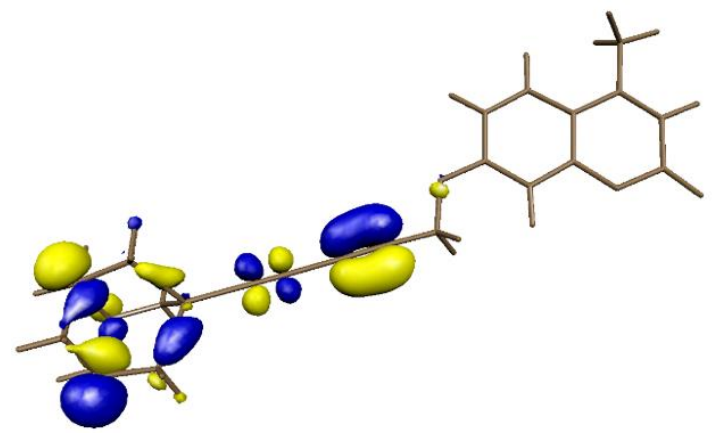

MO 105: $-0.240 \mathrm{Ha}$

PTA (51), CC (31), Au (7)

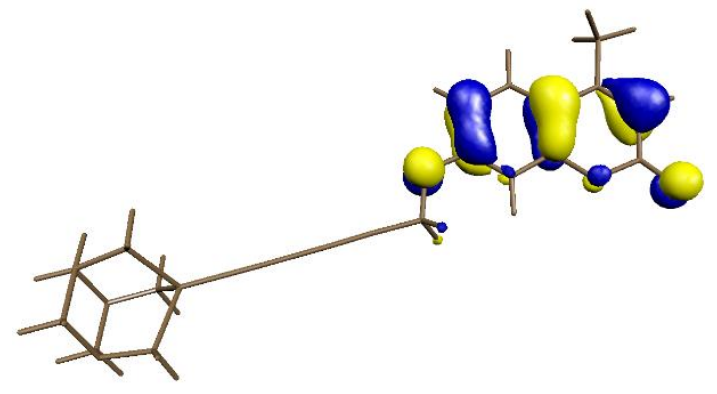

MO 107 (HOMO): $-0.221 \mathrm{Ha}$

Cou (80), $\mathrm{OCH}_{2}(14)$

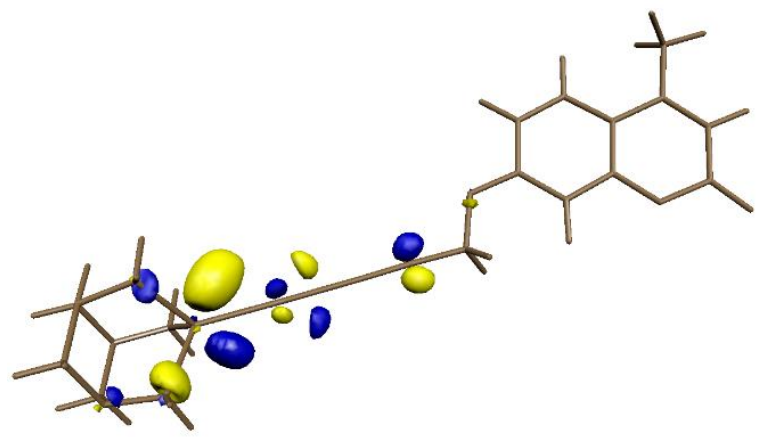

MO 109: $-0.039 \mathrm{Ha}$

PTA (61), Au (42), CC (8)

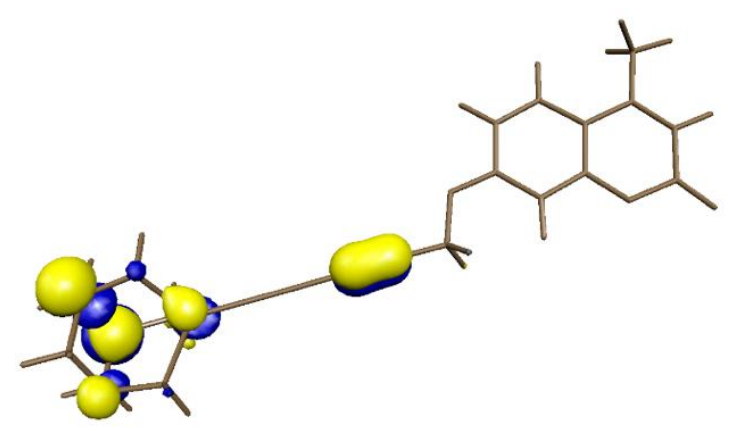

MO 104: $-0.248 \mathrm{Ha}$

PTA (69), CC (20)

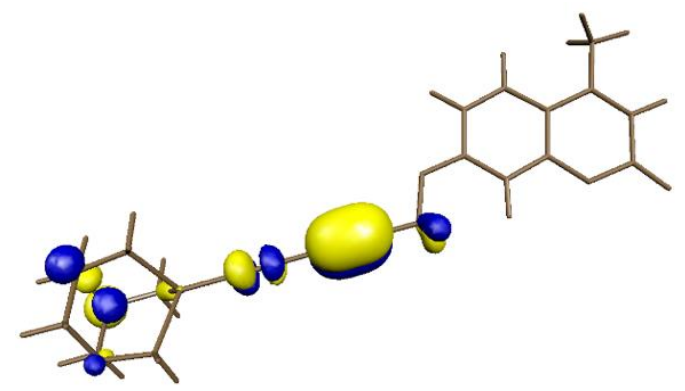

MO 106: -0.235 Ha

CC (60), PTA (17), Au (8), $\mathrm{OCH}_{2}$ (4)

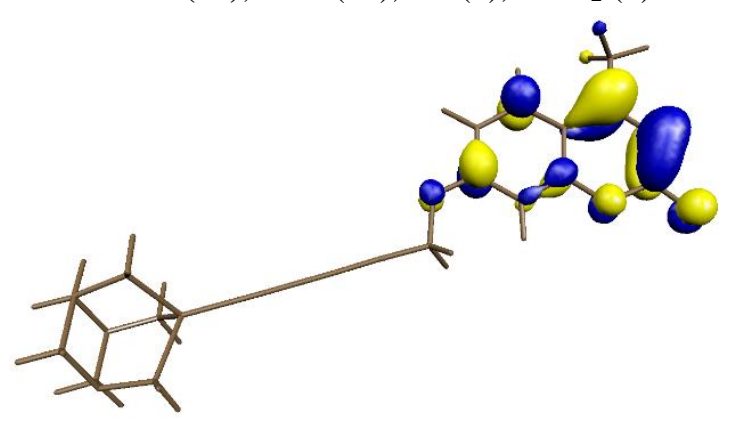

MO 108 (LUMO): $-0.059 \mathrm{Ha}$

Cou (94), $\mathrm{OCH}_{2}(2)$

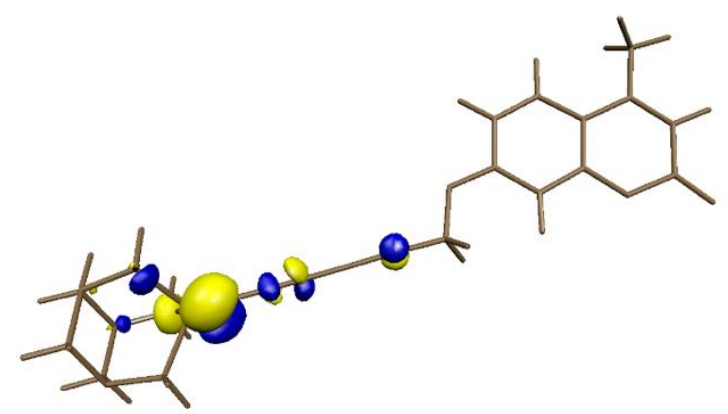

MO 110: $-0.037 \mathrm{Ha}$

PTA (48), Au (42), CC (5)

Figure S12. Molecular orbitals for complex 2a. Percentage compositions for each one entry from Natural Populations Analysis are shown. 


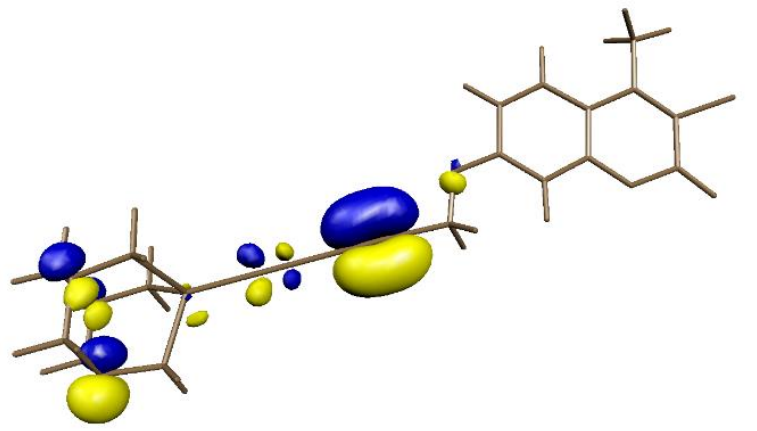

MO 111: $-0.253 \mathrm{Ha}$

CC (56), PTA (32), Au (3)

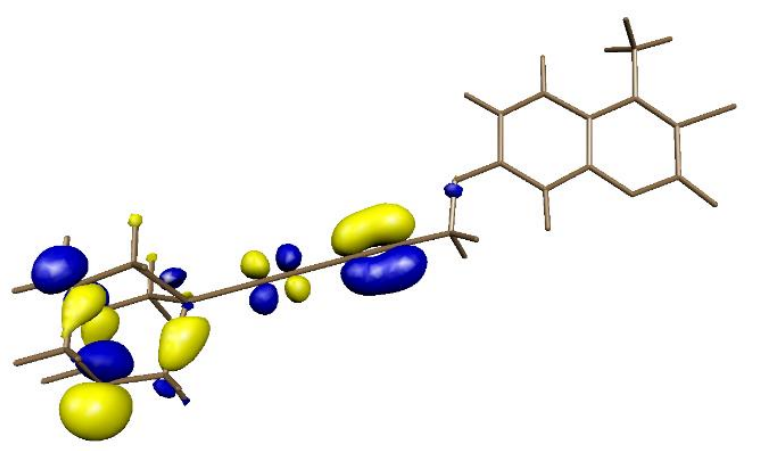

MO 113: $-0.249 \mathrm{Ha}$

PTA (55), CC (28), Au (7)

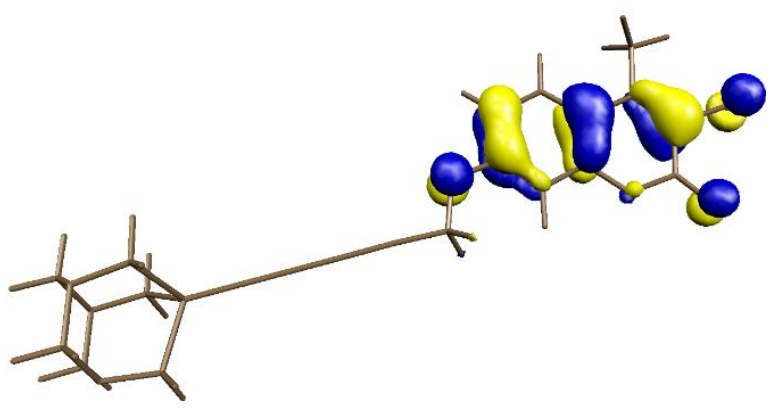

MO 115 (HOMO): $-0.224 \mathrm{Ha}$

Cou (80), $\mathrm{OCH}_{2}(14)$

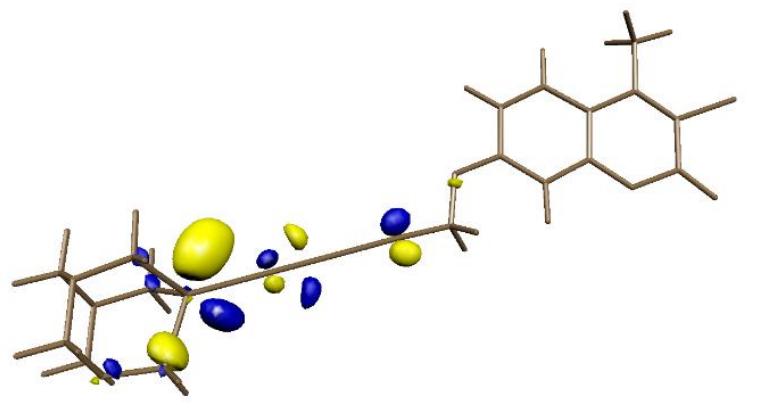

MO 117: $-0.041 \mathrm{Ha}$

PTA (48), Au (42), CC (8)

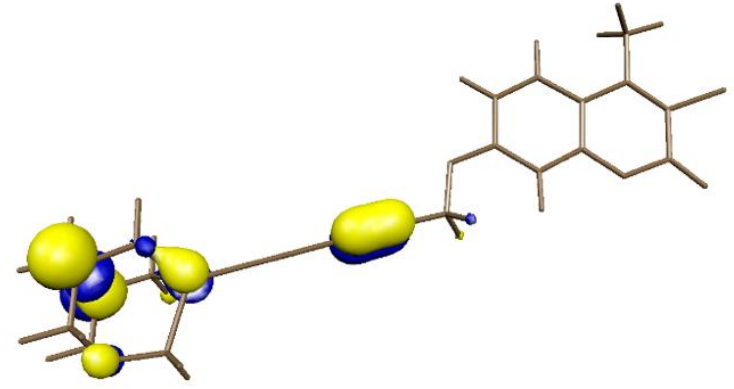

MO 112: $-0.249 \mathrm{Ha}$

PTA (69), CC (23)

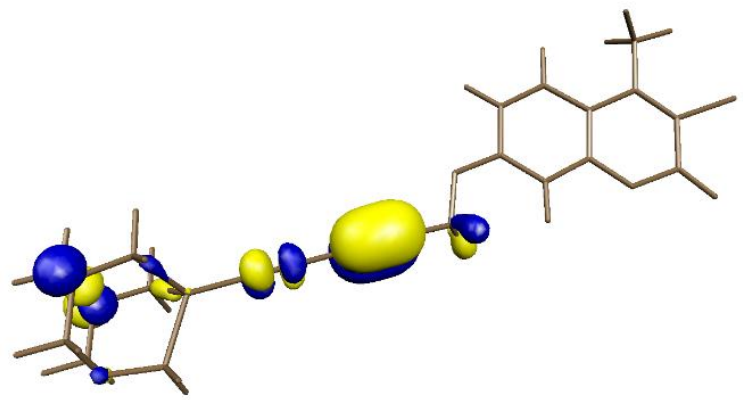

MO 114: $-0.236 \mathrm{Ha}$

CC (58), PTA (22), Au (8), $\mathrm{OCH}_{2}$ (4)

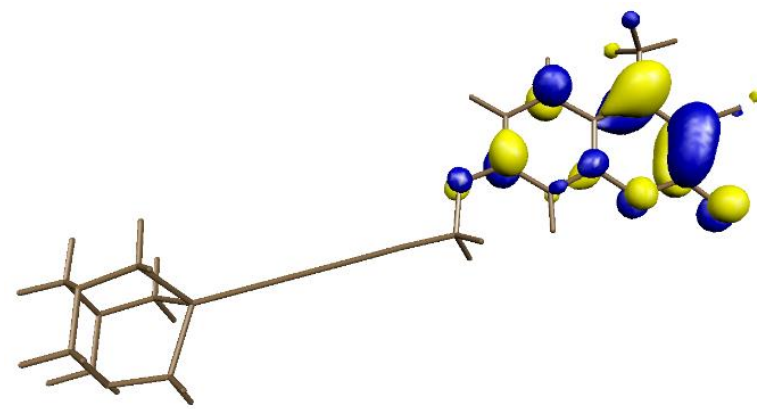

MO 116 (LUMO): $-0.067 \mathrm{Ha}$

Cou (94)

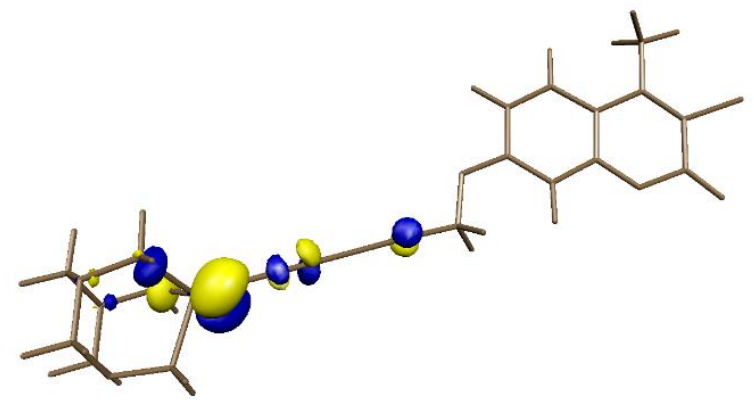

MO 118: $-0.039 \mathrm{Ha}$

PTA (46), Au (42), CC (6)

Figure S13. Molecular orbitals for complex 3a. Percentage compositions for each one entry from Natural Populations Analysis are shown. 


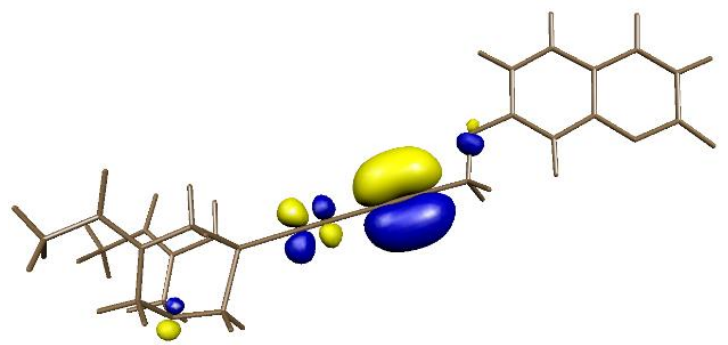

MO 120: $-0.250 \mathrm{Ha}$

CC (79), $\mathrm{Au}(9), \mathrm{OCH}_{2}$ (5)

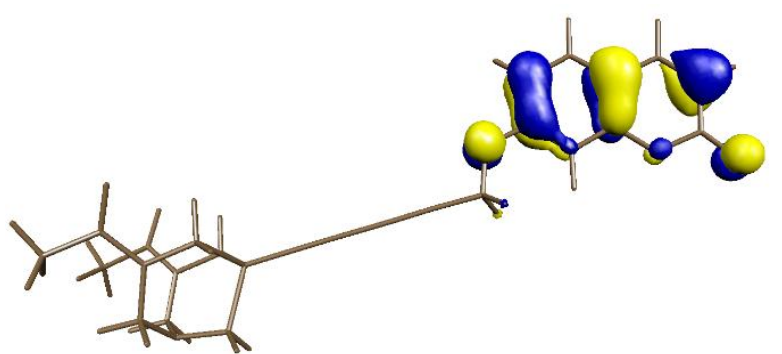

MO 122 (HOMO): $-0.224 \mathrm{Ha}$

Cou (82), $\mathrm{OCH}_{2}(14)$

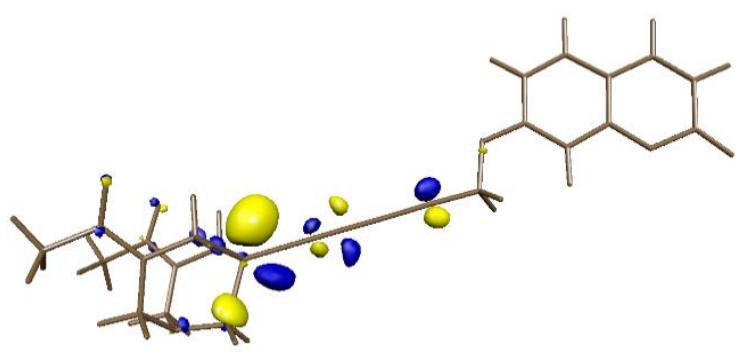

MO 124: $-0.045 \mathrm{Ha}$

DAPTA (50), Au (36), CC (7)

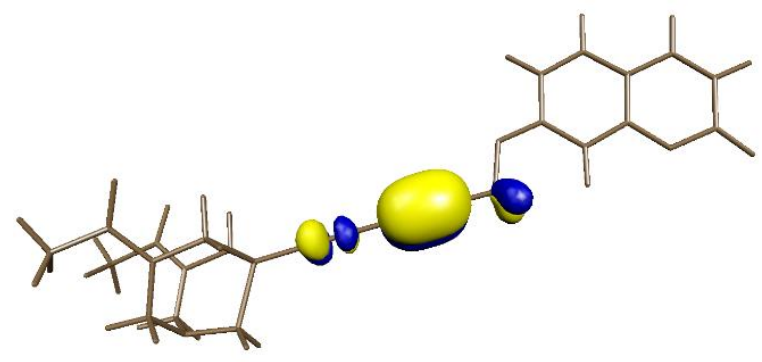

MO 121: $-0.241 \mathrm{Ha}$

CC (80), $\mathrm{Au}(8), \mathrm{OCH}_{2}(5)$

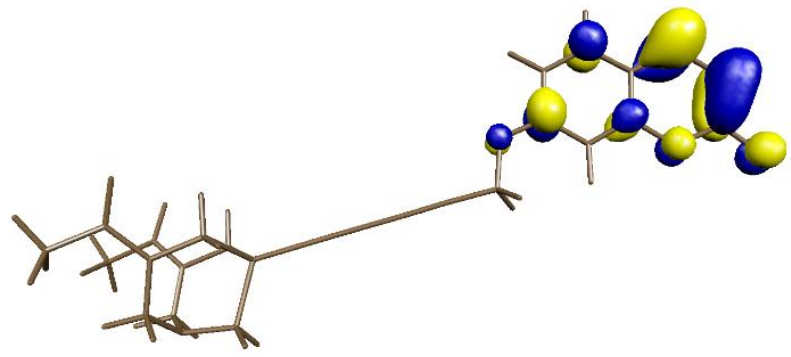

MO 123 (LUMO): $-0.064 \mathrm{Ha}$

Cou (94)

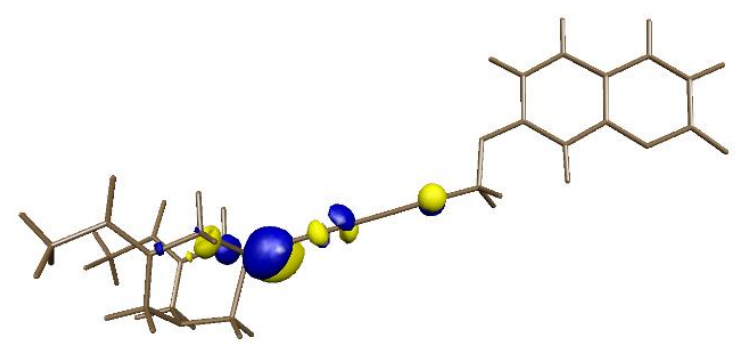

MO 125: $-0.039 \mathrm{Ha}$

DAPTA (44), Au (43), CC (7)

Figure S14. Molecular orbitals for complex 1b. Percentage compositions for each one entry from Natural Populations Analysis are shown. 


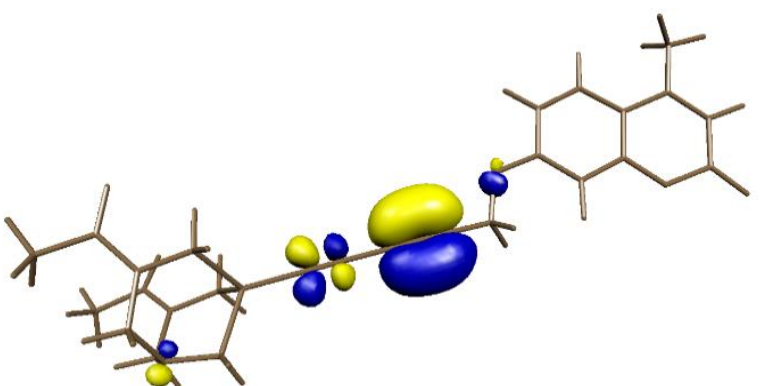

MO 124: $-0.249 \mathrm{Ha}$

CC (79), $\mathrm{Au}(9), \mathrm{OCH}_{2}$ (4)

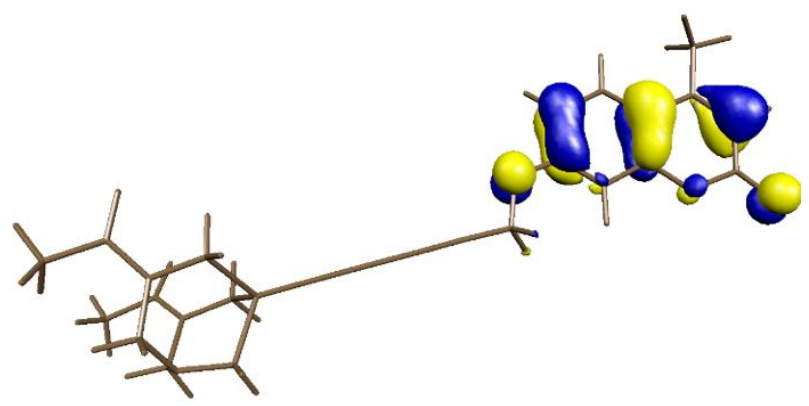

MO 126 (HOMO): $-0.222 \mathrm{Ha}$

Cou (82), $\mathrm{OCH}_{2}$ (14)

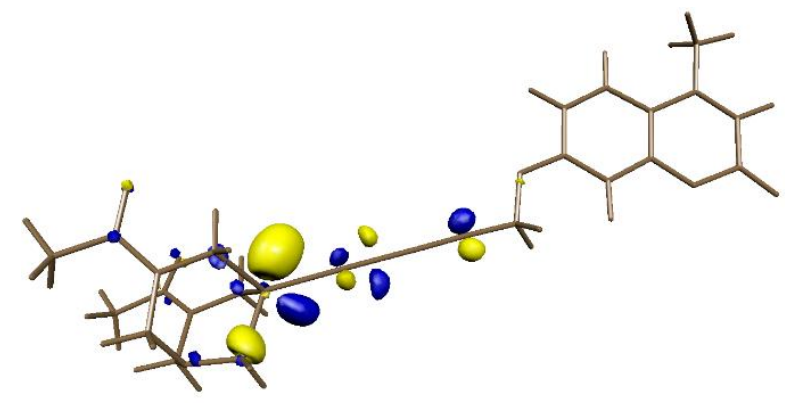

MO 128: -0.044 Ha

DAPTA (51), Au (36), CC (7)

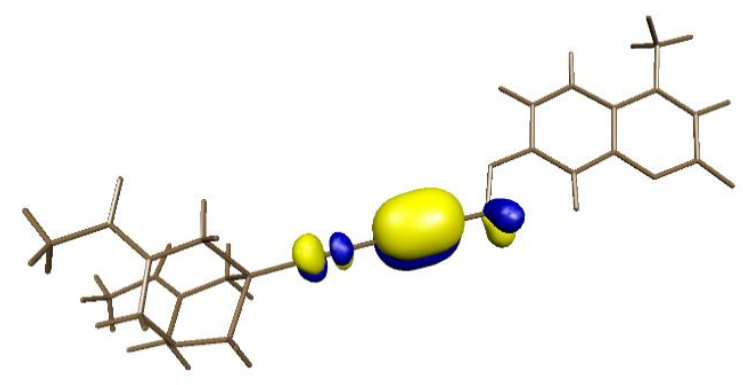

MO 125: $-0.240 \mathrm{Ha}$

CC (80), $\mathrm{Au}(8), \mathrm{OCH}_{2}(5)$

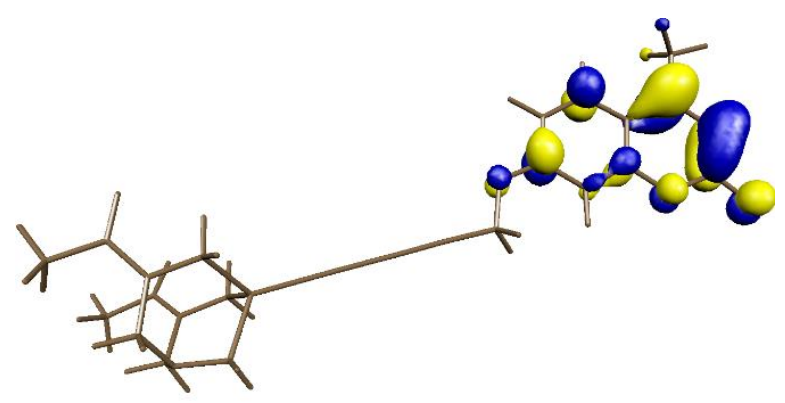

MO 127 (LUMO): $-0.059 \mathrm{Ha}$

Cou (93)

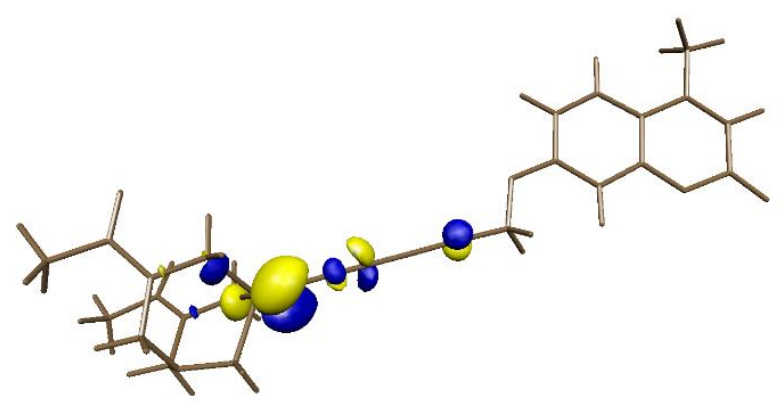

MO 129: $-0.039 \mathrm{Ha}$

DAPTA (43), Au (43), CC (6)

Figure S15. Molecular orbitals for complex 2b. Percentage compositions for each one entry from Natural Populations Analysis are shown. 


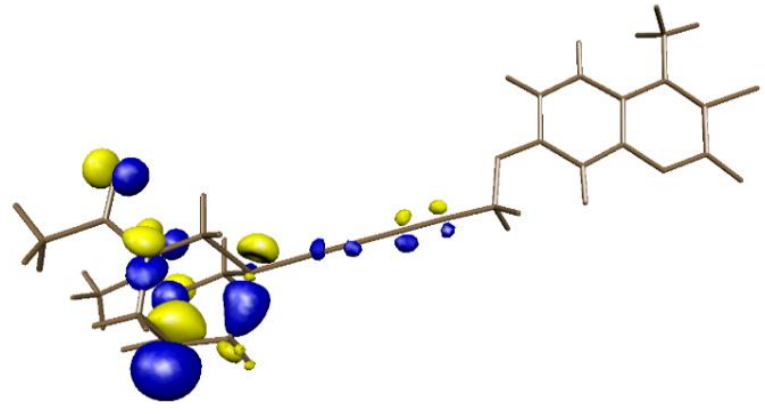

MO 129: $-0.274 \mathrm{Ha}$

DAPTA (79), CC (4)

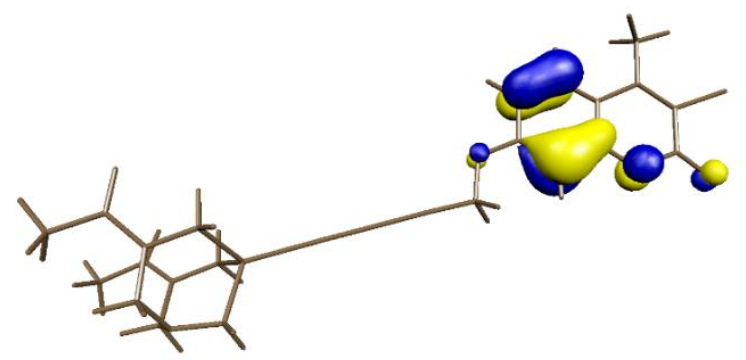

MO 131: $-0.259 \mathrm{Ha}$

Cou (93)

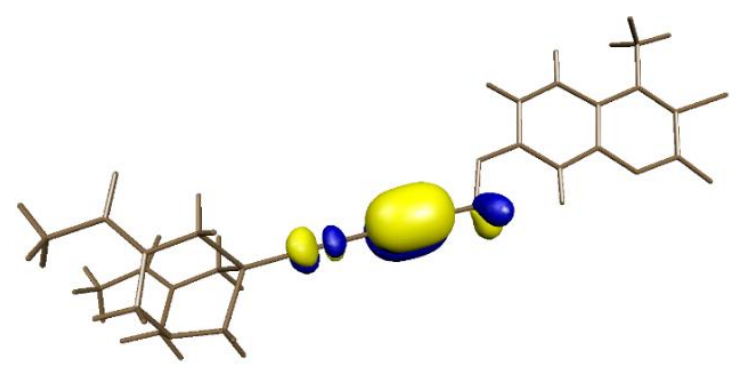

MO 133: $-0.242 \mathrm{Ha}$

CC (80), Au (7), $\mathrm{OCH}_{2}$ (5)

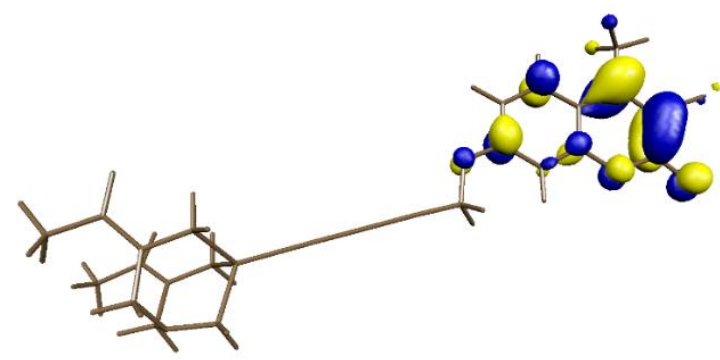

MO 135 (LUMO): $-0.067 \mathrm{Ha}$

Cou (94)

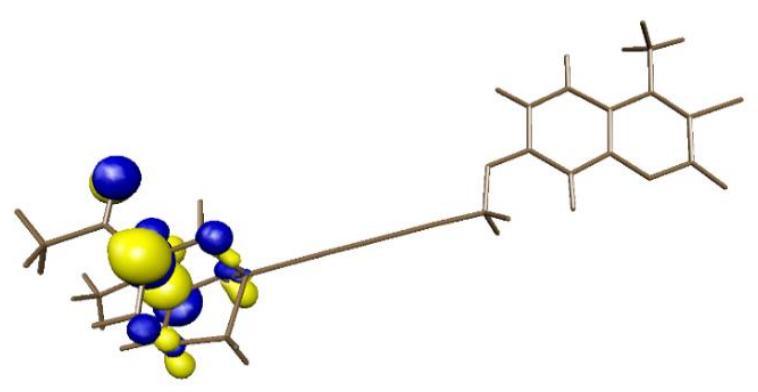

MO 130: -0.269 Ha

DAPTA (93)

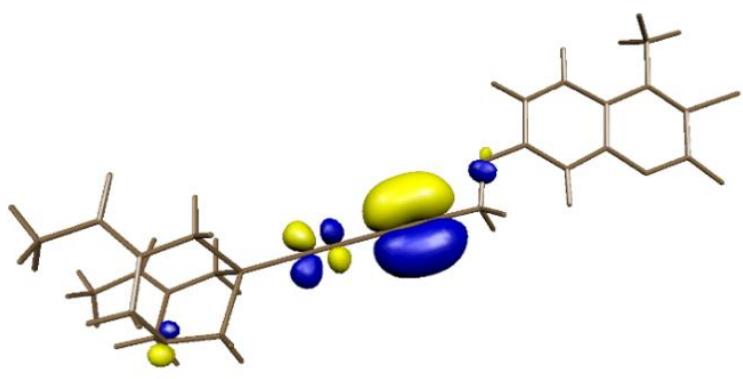

MO 132: -0.251 Ha

CC (79), $\mathrm{Au}(9), \mathrm{OCH}_{2}$ (4)

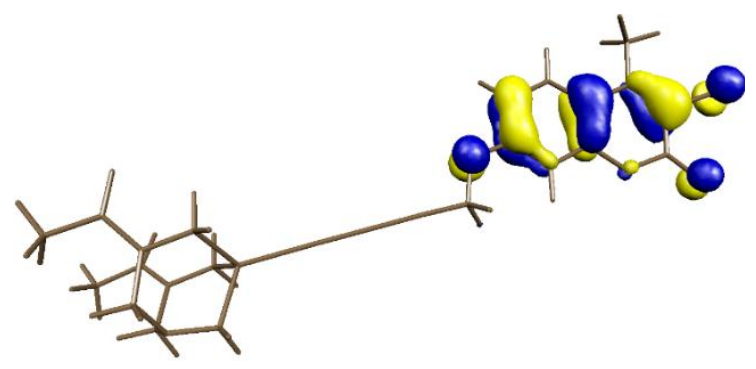

MO 134 (HOMO): $-0.224 \mathrm{Ha}$

Cou (81), $\mathrm{OCH}_{2}$ (11)

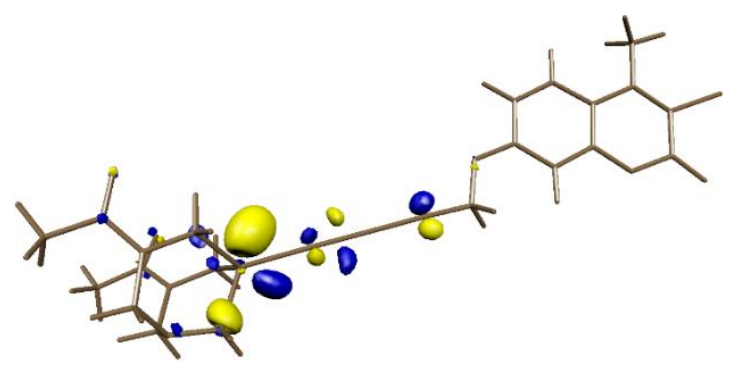

MO 136: $-0.046 \mathrm{Ha}$

DAPTA (51), Au (36), CC (7) 


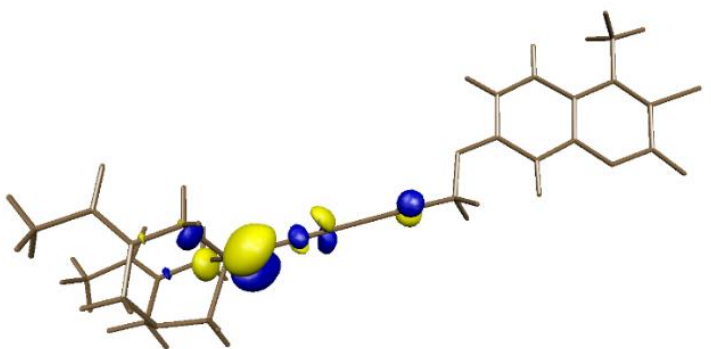

MO 137: $-0.040 \mathrm{Ha}$

DAPTA (44), Au (43), CC (7)

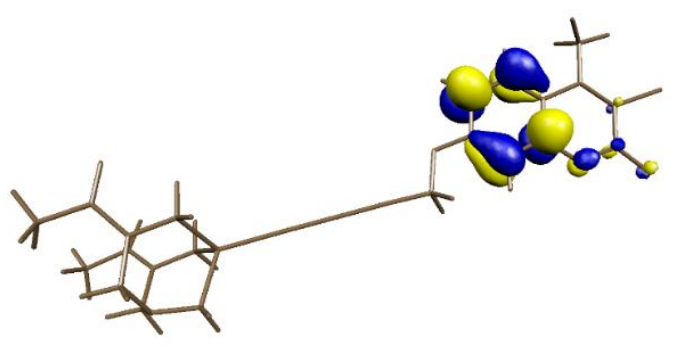

MO 139: $-0.022 \mathrm{Ha}$

Cou (92)

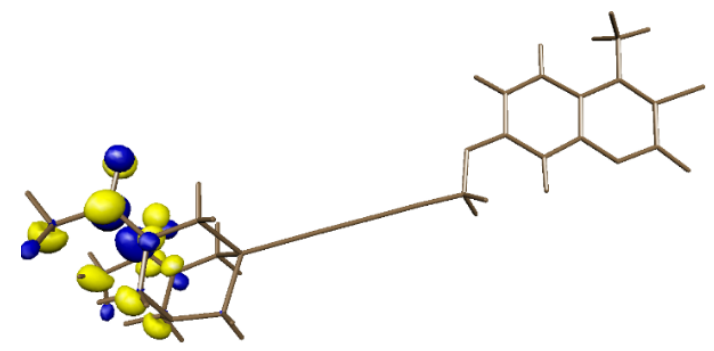

MO 141: $-0.008 \mathrm{Ha}$

DAPTA (75)

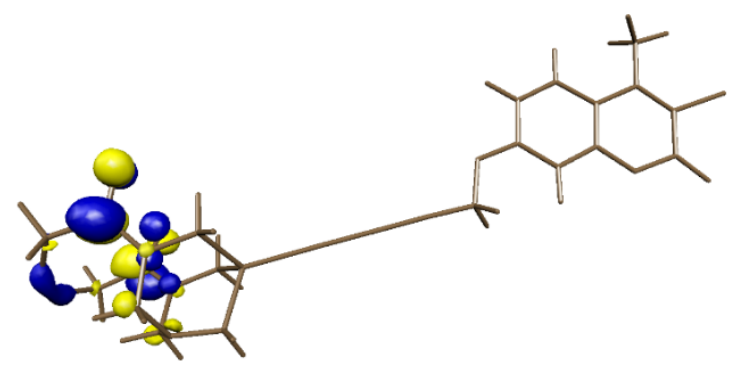

MO 138: $-0.026 \mathrm{Ha}$

DAPTA (71), Au (5)

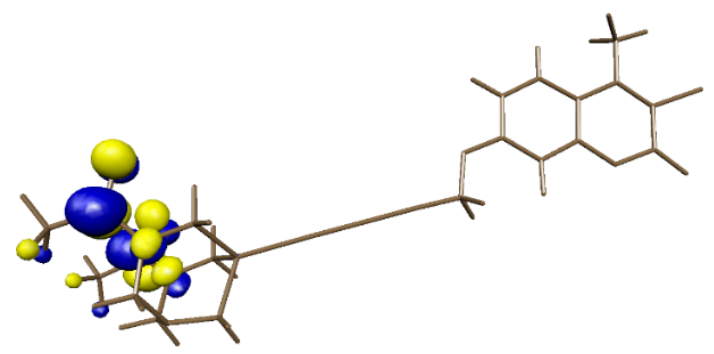

MO 140: $-0.010 \mathrm{Ha}$

DAPTA (94)

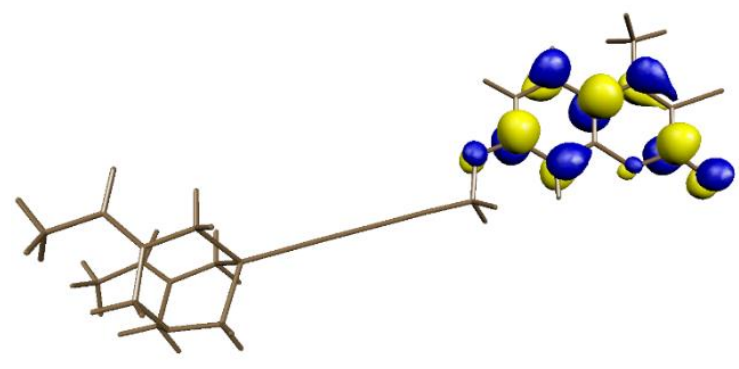

MO 142: $-0.000 \mathrm{Ha}$

Cou (90)

Figure S16. Molecular orbitals for complex 3b. Percentage compositions for each one entry from Natural Populations Analysis are shown. 


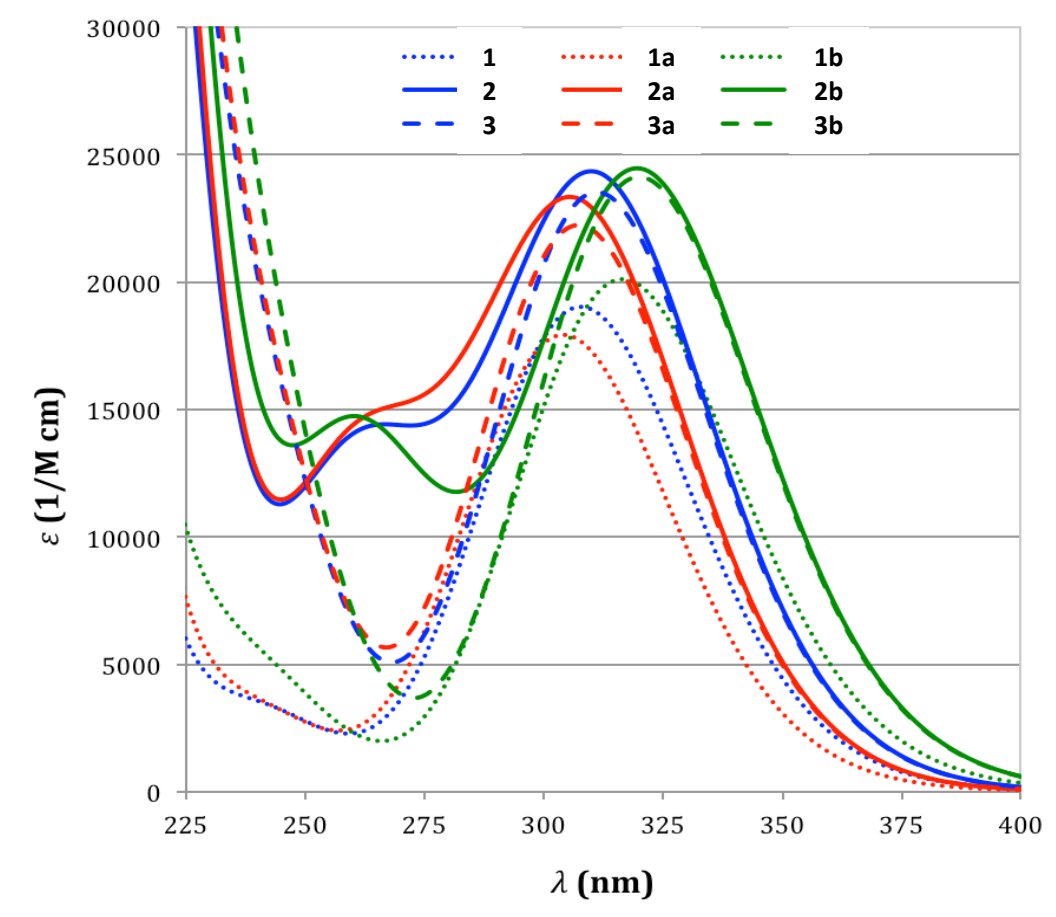

Figure S17. Calculated absorption spectra of 1-3 and respective gold(I) complexes in acetonitrile solution. 
Table S2. Calculated absorption parameters (wavelengths in $\mathrm{nm}$ and their intensities) for gold coumarin derivatives in gas phase and acetonitrile solution. For each entry, main contributions of the orbitals for the transition and their coefficients are shown.

(a) Complex 1a

\begin{tabular}{|c|c|c|c|}
\hline \multicolumn{2}{|r|}{ Gas phase } & \multicolumn{2}{|r|}{$\mathrm{CH}_{3} \mathrm{CN}$} \\
\hline$\lambda(f)$ & Assignment & $\lambda(f)$ & Assignment \\
\hline $308(0.49)$ & $\begin{array}{l}\text { Coumarin: } \\
\pi(\mathrm{Cou}) \rightarrow \pi^{*}(\mathrm{Cou})[94 \%]\end{array}$ & $312(0.57)$ & $\begin{array}{l}\text { Coumarin: } \\
\pi(\mathrm{Cou}) \rightarrow \pi^{*}(\mathrm{Cou}) \quad[97 \%]\end{array}$ \\
\hline $253(0.33)$ & $\begin{array}{l}\text { Intraligand: } \\
\mathrm{PTA}+\pi(\mathrm{CC})_{\|} \rightarrow \mathrm{PTA}+p(\mathrm{Au})[56 \%] \\
\pi(\mathrm{CC})+\mathrm{PTA} \rightarrow \mathrm{PTA}+p(\mathrm{Au})[23 \%] \\
\pi(\mathrm{CC})+\mathrm{PTA} \rightarrow \mathrm{PTA}+p(\mathrm{Au})[17 \%]\end{array}$ & $263(0.27)$ & $\begin{array}{l}\text { Intraligand: } \\
\mathrm{PTA} \rightarrow \mathrm{PTA}+p(\mathrm{Au})[56 \%] \\
\mathrm{PTA} \rightarrow p(\mathrm{Au})+\mathrm{PTA}[28 \%] \\
\mathrm{PTA} \rightarrow \pi^{*}(\mathrm{Cou})+p(\mathrm{Au})+\mathrm{PTA}[14 \%]\end{array}$ \\
\hline
\end{tabular}

(b) Complex 2a

\begin{tabular}{|c|c|c|c|}
\hline \multicolumn{2}{|r|}{ Gas phase } & \multicolumn{2}{|r|}{$\mathrm{CH}_{3} \mathrm{CN}$} \\
\hline$\lambda(f)$ & Assignment & $\lambda(f)$ & Assignment \\
\hline $306(0.47)$ & $\begin{array}{l}\text { Coumarin: } \\
\pi(\mathrm{Cou}) \rightarrow \pi^{*}(\mathrm{Cou})[94 \%]\end{array}$ & $307(0.46)$ & $\begin{array}{l}\text { Coumarin: } \\
\pi(\mathrm{Cou}) \rightarrow \pi^{*}(\mathrm{Cou})[82 \%] \\
\mathrm{PTA} \rightarrow \pi^{*}(\mathrm{Cou})[15 \%]\end{array}$ \\
\hline $253(0.35)$ & $\begin{array}{l}\text { Intraligand: } \\
\mathrm{PTA}+\pi(\mathrm{CC})_{\|} \rightarrow \mathrm{PTA}+p(\mathrm{Au})[56 \%] \\
\pi(\mathrm{CC})+\mathrm{PTA} \rightarrow \mathrm{PTA}+p(\mathrm{Au})[22 \%] \\
\pi(\mathrm{CC})+\mathrm{PTA} \rightarrow \mathrm{PTA}+p(\mathrm{Au})[18 \%]\end{array}$ & $262(0.27)$ & $\begin{array}{l}\text { Intraligand: } \\
\mathrm{PTA} \rightarrow \mathrm{PTA}+p(\mathrm{Au})[61 \%] \\
\mathrm{PTA} \rightarrow p(\mathrm{Au})+\mathrm{PTA}[28 \%] \\
\mathrm{PTA} \rightarrow \pi^{*}(\mathrm{Cou})+p(\mathrm{Au})+\mathrm{PTA}[9 \%]\end{array}$ \\
\hline
\end{tabular}

(c) Complex 3a

\begin{tabular}{|c|c|c|c|}
\hline \multicolumn{2}{|r|}{ Gas phase } & \multicolumn{2}{|r|}{$\mathrm{CH}_{3} \mathrm{CN}$} \\
\hline$\lambda(f)$ & Assignment & $\lambda(f)$ & Assignment \\
\hline $316(0.53)$ & $\begin{array}{l}\text { Coumarin: } \\
\pi(\mathrm{Cou}) \rightarrow \pi^{*}(\mathrm{Cou})[95 \%]\end{array}$ & $320(0.57)$ & $\begin{array}{l}\text { Coumarin: } \\
\pi(\mathrm{Cou}) \rightarrow \pi^{*}(\mathrm{Cou})[93 \%]\end{array}$ \\
\hline $253(0.37)$ & $\begin{array}{l}\text { Intraligand: } \\
\text { PTA }+\pi(\mathrm{CC}) \rightarrow \mathrm{PTA}+p(\mathrm{Au})[58 \%] \\
\pi(\mathrm{CC})+\mathrm{PTA} \rightarrow \mathrm{PTA}+p(\mathrm{Au})[23 \%] \\
\pi(\mathrm{CC})+\mathrm{PTA} \rightarrow \mathrm{PTA}+p(\mathrm{Au})[16 \%]\end{array}$ & $263(0.28)$ & $\begin{array}{l}\text { Intraligand: } \\
\mathrm{PTA} \rightarrow \mathrm{PTA}+p(\mathrm{Au})[46 \%] \\
\mathrm{PTA} \rightarrow p(\mathrm{Au})+\mathrm{PTA}[28 \%] \\
\mathrm{PTA} \rightarrow \mathrm{PTA}+p(\mathrm{Au})[23 \%]\end{array}$ \\
\hline
\end{tabular}


(d) Complex $\mathbf{1 b}$

\begin{tabular}{|c|c|c|c|}
\hline \multicolumn{2}{|r|}{ Gas phase } & \multicolumn{2}{|r|}{$\mathrm{CH}_{3} \mathrm{CN}$} \\
\hline$\lambda(f)$ & Assignment & $\lambda(f)$ & Assignment \\
\hline $308(0.49)$ & $\begin{array}{l}\text { Coumarin: } \\
\pi(\mathrm{Cou}) \rightarrow \pi^{*}(\mathrm{Cou})[94 \%]\end{array}$ & $312(0.57)$ & $\begin{array}{l}\text { Coumarin: } \\
\pi(\mathrm{Cou}) \rightarrow \pi^{*}(\mathrm{Cou})[97 \%]\end{array}$ \\
\hline $239(0.73)$ & $\begin{array}{l}\text { Intraligand: } \\
\pi(\mathrm{CC}) \rightarrow \pi^{*}(\mathrm{Cou})[52 \%] \\
\pi(\mathrm{CC}) \rightarrow \mathrm{PTA}+p(\mathrm{Au})[39 \%]\end{array}$ & $237(0.14)$ & $\begin{array}{l}\text { Intraligand: } \\
\mathrm{PTA} \rightarrow \pi^{*}(\mathrm{Cou})[22 \%] \\
\pi(\mathrm{Cou}) \rightarrow \mathrm{PTA}+p(\mathrm{Au})[21 \%] \\
\mathrm{PTA} \rightarrow \pi^{*}(\mathrm{Cou})[10 \%] \\
\mathrm{PTA} \rightarrow \mathrm{PTA}+p(\mathrm{Au})[10 \%] \\
\pi(\mathrm{Cou}) \rightarrow \mathrm{PTA}+p(\mathrm{Au})[9 \%]\end{array}$ \\
\hline
\end{tabular}

(e) Complex 2b

\begin{tabular}{|c|c|c|c|}
\hline \multicolumn{2}{|r|}{ Gas phase } & \multicolumn{2}{|r|}{$\mathrm{CH}_{3} \mathrm{CN}$} \\
\hline$\lambda(f)$ & Assignment & $\lambda(f)$ & Assignment \\
\hline $305(0.47)$ & $\begin{array}{l}\text { Coumarin: } \\
\pi(\mathrm{Cou}) \rightarrow \pi^{*}(\mathrm{Cou})[94 \%]\end{array}$ & $308(0.54)$ & $\begin{array}{l}\text { Coumarin: } \\
\pi(\mathrm{Cou}) \rightarrow \pi^{*}(\mathrm{Cou})[96 \%]\end{array}$ \\
\hline $240(0.74)$ & $\begin{array}{l}\text { Intraligand: } \\
\pi(\mathrm{CC}) \rightarrow \text { PTA }+p(\mathrm{Au})[50 \%] \\
\pi(\mathrm{CC}) \rightarrow \text { PTA }+p(\mathrm{Au})[38 \%]\end{array}$ & $237(0.18)$ & $\begin{array}{l}\text { Intraligand: } \\
\pi(\mathrm{Cou}) \rightarrow \pi^{*}(\mathrm{Cou})+\mathrm{PTA}+p(\mathrm{Au})[33 \%] \\
\mathrm{PTA} \rightarrow \mathrm{PTA}+p(\mathrm{Au})[19 \%] \\
\pi(\mathrm{Cou}) \rightarrow \mathrm{PTA}+p(\mathrm{Au})[14 \%] \\
\pi(\mathrm{CC}) \rightarrow \mathrm{PTA}+p(\mathrm{Au})[10 \%]\end{array}$ \\
\hline
\end{tabular}

\section{(f) Complex 3b}

\begin{tabular}{|c|c|c|c|}
\hline \multicolumn{2}{|r|}{ Gas phase } & \multicolumn{2}{|r|}{$\mathrm{CH}_{3} \mathrm{CN}$} \\
\hline$\lambda(f)$ & Assignment & $\lambda(f)$ & Assignment \\
\hline $315(0.54)$ & $\begin{array}{l}\text { Coumarin: } \\
\pi(\mathrm{Cou}) \rightarrow \pi^{*}(\mathrm{Cou})[95 \%]\end{array}$ & $320(0.59)$ & $\begin{array}{l}\text { Coumarin: } \\
\pi(\mathrm{Cou}) \rightarrow \pi^{*}(\mathrm{Cou})[97 \%]\end{array}$ \\
\hline $247(0.06)$ & $\begin{array}{l}\text { Coumarin: } \\
\pi(\mathrm{Cou}) \rightarrow \pi^{*}(\mathrm{Cou})[67 \%] \\
\pi(\mathrm{Cou}) \rightarrow \pi^{*}(\mathrm{Cou})[21 \%]\end{array}$ & $247(0.10)$ & $\begin{array}{l}\text { Coumarin: } \\
\pi(\mathrm{Cou}) \rightarrow \mathrm{PTA}+p(\mathrm{Au})[59 \%] \\
\pi(\mathrm{Cou}) \rightarrow \pi^{*}(\mathrm{Cou})[12 \%] \\
\pi(\mathrm{Cou}) \rightarrow \mathrm{PTA}+p(\mathrm{Au})[9 \%]\end{array}$ \\
\hline $239(0.79)$ & $\begin{array}{l}\text { Intraligand: } \\
\pi(\mathrm{CC}) \rightarrow \mathrm{PTA}+p(\mathrm{Au})[50 \%] \\
\pi(\mathrm{CC}) \rightarrow \mathrm{PTA}+p(\mathrm{Au})[38 \%]\end{array}$ & $237(0.19)$ & $\begin{array}{l}\text { Intraligand: } \\
\pi(\mathrm{Cou}) \rightarrow \mathrm{PTA}+p(\mathrm{Au})[27 \%] \\
\pi(\mathrm{Cou}) \rightarrow \mathrm{PTA}+p(\mathrm{Au})[23 \%] \\
\mathrm{PTA} \rightarrow \mathrm{PTA}+p(\mathrm{Au})[11 \%] \\
\pi(\mathrm{CC}) \rightarrow \mathrm{PTA}+p(\mathrm{Au})[9 \%] \\
\pi(\mathrm{CC}) \rightarrow \mathrm{PTA}+p(\mathrm{Au})[8 \%]\end{array}$ \\
\hline
\end{tabular}


Table S3. Main geometrical parameters for optimized structures for Coum- $\mathrm{H}$ and its complexes.

(a) Optimization as ground singlet state $\left(S_{0}\right)$

\begin{tabular}{|c|c|c|c|c|c|c|c|c|}
\hline Comp & $C^{10}-C^{4}$ & $C^{4}-C^{3}$ & $C^{3}-C^{2}$ & $\mathrm{C}^{2}-\mathrm{O}^{1}$ & $\mathbf{C}^{2}=\mathbf{O}$ & $\mathrm{C}^{3}-\mathrm{Cl}$ & $\Sigma \mathbf{C}^{\mathbf{3}}$ & $\theta\left(\mathbf{C}^{4} \cdot \mathbf{O}^{1}\right)$ \\
\hline 1 & 1.43 & 1.35 & 1.45 & 1.40 & 1.20 & - & 360.0 & 180.0 \\
\hline $1 \mathrm{a}$ & 1.43 & 1.35 & 1.45 & 1.40 & 1.20 & - & 360.0 & 180.0 \\
\hline $1 b$ & 1.43 & 1.35 & 1.45 & 1.40 & 1.20 & - & 360.0 & 180.0 \\
\hline 2 & 1.44 & 1.35 & 1.45 & 1.40 & 1.20 & - & 360.0 & 180.0 \\
\hline $2 a$ & 1.45 & 1.35 & 1.45 & 1.40 & 1.20 & - & 360.0 & 180.0 \\
\hline $2 b$ & 1.45 & 1.35 & 1.45 & 1.40 & 1.20 & - & 360.0 & 180.0 \\
\hline 3 & 1.45 & 1.36 & 1.47 & 1.39 & 1.20 & 1.75 & 360.0 & 180.0 \\
\hline $3 a$ & 1.45 & 1.36 & 1.46 & 1.39 & 1.20 & 1.75 & 360.0 & 180.0 \\
\hline $3 b$ & 1.45 & 1.36 & 1.46 & 1.39 & 1.20 & 1.75 & 360.0 & 180.0 \\
\hline
\end{tabular}


(b) Optimization as first triplet state $\left(T_{1}\right)$

\begin{tabular}{|c|c|c|c|c|c|c|c|c|}
\hline Comp & $C^{10}-C^{4}$ & $C^{4}-C^{3}$ & $C^{3}-C^{2}$ & $C^{2}-O^{1}$ & $\mathbf{C}^{2}=\mathbf{O}$ & $C^{3}-C l$ & $\Sigma \mathbf{C}^{\mathbf{3}}$ & $\theta\left(\mathbf{C}^{4} \cdot \mathbf{O}^{1}\right)$ \\
\hline 1 & 1.38 & 1.45 & 1.42 & 1.41 & 1.21 & - & 360.0 & 179.9 \\
\hline $1 \mathrm{a}$ & 1.38 & 1.45 & 1.42 & 1.41 & 1.22 & - & 360.0 & 180.0 \\
\hline $1 b$ & 1.37 & 1.44 & 1.41 & 1.41 & 1.22 & - & 360.0 & 179.8 \\
\hline 2 & 1.38 & 1.45 & 1.42 & 1.41 & 1.21 & - & 360.0 & 179.7 \\
\hline $2 a$ & 1.39 & 1.45 & 1.42 & 1.41 & 1.22 & - & 360.0 & 179.2 \\
\hline $2 b$ & 1.38 & 1.45 & 1.41 & 1.40 & 1.22 & - & 360.0 & 179.7 \\
\hline 3 & 1.39 & 1.47 & 1.43 & 1.40 & 1.21 & 1.72 & 359.8 & 165.7 \\
\hline $3 a$ & 1.39 & 1.47 & 1.43 & 1.40 & 1.21 & 1.73 & 359.8 & 165.1 \\
\hline $3 \mathbf{b}$ & 1.39 & 1.47 & 1.43 & 1.40 & 1.21 & 1.73 & 359.8 & 165.1 \\
\hline
\end{tabular}


Table S4. Calculated energies for lowest singlet and triplet states $S_{1}$ and $T_{1}$ (in eV, and $\mathrm{nm}$ in parenthesis) from the ground state $S_{0}$ for the coumarin derivatives in acetonitrile solution. For optimized triplet state $T_{1}$, relative energies from the ground state $S_{0}$, and phosphorescence emission $\left(T_{1} \sim>S_{0}\right)$ are also shown.

\begin{tabular}{|c|cc|ccc|}
\hline \multirow{2}{*}{ Comp } & \multicolumn{2}{|c|}{ Optimized Geometry $\boldsymbol{S}_{\mathbf{0}}$} & \multicolumn{3}{|c|}{ Optimized Geometry $\boldsymbol{T}_{\mathbf{1}}$} \\
\cline { 2 - 6 } & $\boldsymbol{S}_{\mathbf{1}}$ & $\boldsymbol{T}_{\mathbf{1}}$ & $\boldsymbol{T}_{\mathbf{1}}$ * & $\boldsymbol{S}_{\mathbf{0}}^{*}$ & Emission \\
\hline $\mathbf{1}$ & $4.02(308)$ & $2.78(446)$ & $2.62(473)$ & 0.30 & $2.15(577)$ \\
$\mathbf{1 a}$ & $3.89(318)$ & $2.76(449)$ & $2.61(475)$ & 0.28 & $2.16(575)$ \\
\hline $\mathbf{1 b}$ & $3.98(312)$ & $2.76(449)$ & $2.61(475)$ & 0.29 & $2.16(575)$ \\
\hline $\mathbf{2 a}$ & $4.07(305)$ & $2.87(432)$ & $2.70(459)$ & 0.31 & $2.22(559)$ \\
$\mathbf{2 b}$ & $4.03(308)$ & $2.85(435)$ & $2.69(460)$ & 0.29 & $2.23(556)$ \\
\hline $\mathbf{3}$ & $3.91(317)$ & $2.74(453)$ & $2.53(489)$ & 0.46 & $1.90(653)$ \\
$\mathbf{3 a}$ & $3.82(325)$ & $2.72(455)$ & $2.53(491)$ & 0.44 & $1.91(649)$ \\
\hline $\mathbf{3 b}$ & $3.87(320)$ & $2.72(455)$ & $2.53(490)$ & 0.44 & $1.91(648)$ \\
\hline
\end{tabular}

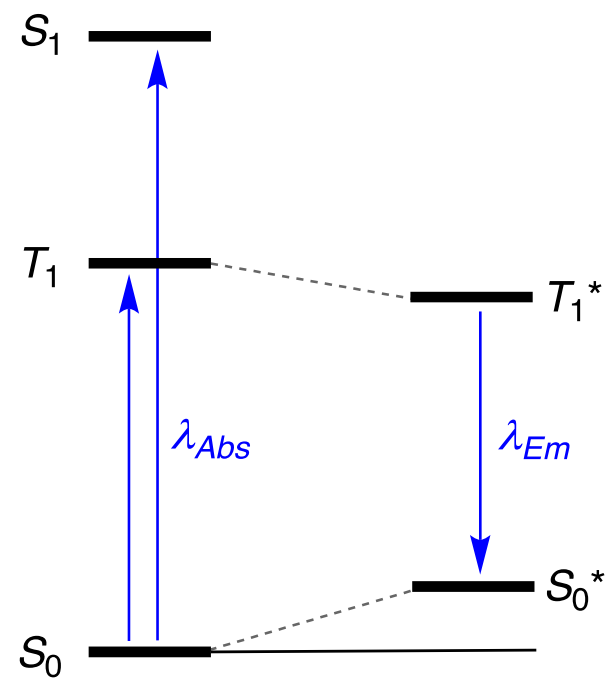

